\title{
Dexmedetomidine exhibits antiarrhythmic effects on human-induced pluripotent stem cell-derived cardiomyocytes through a $\mathrm{Na} / \mathrm{Ca}$ channel-mediated mechanism
}

\author{
Li Yang ${ }^{1 \#}$, Yiqi Gong ${ }^{2 \#}$, Yao Tan $^{2,3}$, Lei Wu ${ }^{4}$, Nevin Witman ${ }^{5}$, Jijian Zheng ${ }^{4}$, Jun Zhang ${ }^{1}$, Wei Fu ${ }^{2,3,6}$, \\ Wei Wang'
}

${ }^{1}$ Department of Anesthesiology, Fudan University Shanghai Cancer Center; Department of Oncology, Shanghai Medical College, Fudan University, Shanghai, China; ${ }^{2}$ Department of Pediatric Cardiothoracic Surgery, Shanghai Children's Medical Center; School of Medicine, Shanghai Jiao Tong University, Shanghai, China; ${ }^{3}$ Institute of Pediatric Translational Medicine, Shanghai Children's Medical Center, School of Medicine, Shanghai Jiao Tong University, Shanghai, China; ${ }^{4}$ Department of Anesthesiology, Shanghai Children's Medical Center; School of Medicine, Shanghai Jiao Tong University, Shanghai, China; ${ }^{5}$ Department of Cell and Molecular Biology, Karolinska Institute, Stockholm, Sweden; ${ }^{6}$ Shanghai Key Laboratory of Tissue Engineering, Shanghai 9th People's Hospital, School of Medicine, Shanghai Jiao Tong University, Shanghai, China

Contributions: (I) Conception and design: W Wang, W Fu, J Zheng; (II) Administrative support: None; (III) Provision of study materials or patients: None; (IV) Collection and assembly of data: L Yang, Y Gong, Y Tan, L Wu; (V) Data analysis and interpretation: L Yang, Y Gong, J Zhang; (VI) Manuscript writing: All authors; (VII) Final approval of manuscript: All authors.

\#These authors contributed equally to this work.

Correspondence to: Wei Fu, PhD. Department of Pediatric Cardiothoracic Surgery, Shanghai Children's Medical Center, School of Medicine, Shanghai Jiao Tong University, 1678 Dong Fang Road, Shanghai 200127, China. Email: fuweizhulu@163.com; Wei Wang, PhD. Department of Pediatric Cardiothoracic Surgery, Shanghai Children's Medical Center, School of Medicine, Shanghai Jiao Tong University, 1678 Dong Fang Road, Shanghai 200127, China. Email: wangwei@scmc.com.cn.

Background: Ventricular-like human-induced pluripotent stem cell-derived cardiomyocytes (hiPSC$\mathrm{CMs}$ ) exhibit the electrophysiological characteristics of spontaneous beating. Previous studies demonstrated that dexmedetomidine (DMED), a highly selective and widely used $\alpha_{2}$-adrenoceptor agonist for sedation, analgesia, and stress management, may induce antiarrhythmic effects, especially ventricular tachycardia. However, the underlying mechanisms of the DMED-mediated antiarrhythmic effects remain to be fully elucidated.

Methods: A conventional patch-clamp recording method was used to investigate the direct effects of DMED on spontaneous action potentials, pacemaker currents $\left(I_{\mathrm{f}}\right)$, potassium $\left(\mathrm{K}^{+}\right)$channel currents $\left(I_{\mathrm{K} 1}\right.$ and $\left.I_{\mathrm{Kr}}\right)$, sodium $\left(\mathrm{Na}^{+}\right)$channel currents $\left(I_{\mathrm{Na}}\right)$, and calcium $\left(\mathrm{Ca}^{2+}\right)$ channel currents $\left(I_{\mathrm{Ca}}\right)$ in ventricular-like hiPSCCMs.

Results: DMED dose-dependently altered the frequency of ventricular-like spontaneous action potentials with a half-maximal inhibitory concentration $\left(\mathrm{IC}_{50}\right)$ of $27.9 \mu \mathrm{M}(\mathrm{n}=6)$ and significantly prolonged the action potential duration at $90 \%$ repolarization $\left(\mathrm{APD}_{90}\right)$. DMED also inhibited the amplitudes of the $I_{\mathrm{Na}}$ and $I_{\mathrm{Ca}}$ without affecting the activation and inactivation curves of these channels. DMED decreased the time constant of the $\mathrm{Na}^{+}$and $\mathrm{Ca}^{2+}$ channel activation at potential -40 to $-20 \mathrm{mv}$, and $-20 \mathrm{mv}$. DMED increased the time constant of inactivation of the $\mathrm{Na}^{+}$and $\mathrm{Ca}^{2+}$ channels. However, DMED did not affect the $I_{\mathrm{K} 1}$, $I_{\mathrm{Kr}}, I_{\mathrm{f}}$, and their current-voltage relationship. The ability of DMED to decrease the spontaneous action potential frequency and the $\mathrm{Na}^{+}$and $\mathrm{Ca}^{2+}$ channel amplitudes, were not blocked by yohimbine, idazoxan, or phentolamine.

\footnotetext{
$\wedge$ ORCID: 0000-0003-2149-7194.
} 
Conclusions: DMED could inhibit the frequency of spontaneous action potentials and decrease the $I_{\mathrm{Na}}$ and $I_{\mathrm{Ca}}$ of hiPSC-CMs via mechanisms that were independent of the $\alpha_{2}$-adrenoceptor, the imidazoline receptor, and the $\alpha_{1}$-adrenoceptor. These inhibitory effects on hiPSC-CMs may contribute to the antiarrhythmic effects of DMED.

Keywords: Dexmedetomidine (DMED); electrophysiology; human-induced pluripotent stem cell-derived cardiomyocytes (hiPSC-CMs); patch clamp recording

Submitted Aug 25, 2020. Accepted for publication Nov 26, 2020.

doi: $10.21037 /$ atm-20-5898

View this article at: http://dx.doi.org/10.21037/atm-20-5898

\section{Introduction}

Human-induced pluripotent stem cell-derived cardiomyocytes (hiPSC-CMs) are among the most promising cell therapy candidates for several heart disease types, including myocardial infarction, which can result in heart failure. Numerous studies have shown that hiPSCCMs can engraft and couple to the host myocardium to contribute to functional improvement (1-3). The expression of physiologically functional ion channels may play an essential role in this functional recovery process $(4,5)$. Since hiPSC-CMs represent a potentially unlimited source of human cardiomyocytes with limited ethical concerns, they may offer an excellent opportunity to explore the physiological and pathophysiological mechanisms that drive human heart function. Furthermore, ventricularlike hiPSC-CMs exhibit the normal electrophysiological characteristics of spontaneous contractions. Therefore, they are a suitable model for studying the physiological effects and mechanisms of certain substances on cardiac tissues at the cellular level $(6,7)$.

Dexmedetomidine (DMED) is a highly selective and potent $\alpha_{2}$-adrenoceptor agonist with sedative, analgesic, and sympatholytic properties (8). The use of DMED is becoming increasingly popular in both pediatric and adult surgeries due to the absence of concurrent respiratory depression and opioid-sparing effects. Although DMED is not well tolerated hemodynamically and increased administrations have been shown to cause hypotension and bradycardia (9-11), these effects are generally clinically benign and self-limiting. DMED has even been shown to have a preventive effect on some types of atrioventricular tachycardia and junctional tachyarrhythmias (12). These antiarrhythmic effects of DMED are believed to be associated with the classical negative feedback mechanism modulating the catecholamine release through the activation of presynaptic $\alpha_{2 \mathrm{~A}}$-adrenoceptors expressed throughout the central nervous system (13-15). However, a study led by Pan et al. (16) revealed that DMED directly inhibited the electrophysiological effects of pacemaker cells in the sinoatrial nodes of rabbits, and this effect was independent of the $\alpha_{2}$-adrenoreceptor. Furthermore, Knaus et al. (17) reported that clonidine, as an $\alpha_{2}$-adrenoreceptor agonist, displayed direct inhibition of cardiac hyperpolarizationactivated cyclic nucleotide-gated (HCN) pacemaker channels contributing to its bradycardic effects in the isolated spontaneously beating right atria of mice. However, the mechanisms by which DMED can directly impact spontaneously beating ventricular cardiomyocytes in humans remain elusive.

The present study aimed to investigate the electrophysiological effects and potential mechanisms of DMED on hiPSC-CMs. Herein, we report the effects of DMED on spontaneous action potentials, potassium $\left(\mathrm{K}^{+}\right)$ channel currents $\left(I_{\mathrm{K} 1}\right.$ and $\left.I_{\mathrm{Kr}}\right)$, hyperpolarization-activated pacemaker currents $\left(I_{\mathrm{f}}\right)$, sodium $\left(\mathrm{Na}^{+}\right)$channel currents $\left(I_{\mathrm{Na}}\right)$, and calcium $\left(\mathrm{Ca}^{2+}\right)$ channel currents $\left(I_{\mathrm{Ca}}\right)$ in hiPSC-CMs over 30 days. The goal was to explore the possible short effectively- and long-term mechanisms of cardiac inhibition by DMED. This article is presented following the Materials Design Analysis Reporting (MDAR) checklist (available at http://dx.doi.org/10.21037/atm-20-5898).

\section{Methods}

\section{The generation and identification of hiPSC-CMs}

The hiPSC-CMs were generated from human-induced pluripotent stem cells (hiPSCs), which were gifted by Professor Li Yanxin of the Shanghai Children's Medical Center. The ethics committee approved all experiments of the Shanghai Children's Medical Center, Shanghai 
Jiao Tong University (SCMCIRB-W2020007). The hiPSCs were generated as previously described (18). Briefly, episomal vectors of octamer-binding transcription factor 4 (OCT4), SRY (sex-determining region Y)-box 2 (SOX2), Kruppel-like factor 4 (KLF4), and MYC were electrotransferred into human cord blood cells to generate the hiPSCs. Cells were maintained in a Matrigel-coated system and cultured with E8 medium (05990, STEMCELL Technologies, Canada). Two days before differentiation, the hiPSCs were dissociated into single cells with Accutase (7920, STEMCELL Technologies, Canada) at $37^{\circ} \mathrm{C}$ for 4 minutes and seeded into an evenly distributed monolayer onto a Matrigel-coated cell culture dish at 100,000-200,000 cell/ $/ \mathrm{cm}^{2}$. On day 0 , hiPSCs were fully confluent and treated with the glycogen synthase kinase 3 (GSK3) selective inhibitor CHIR99021 (72054, STEMCELL Technologies, Canada) in Roswell Park Memorial Institute (RPMI) 1640/B27 medium without insulin (RPMI1640/B27-ins, GIBCO, 11875093/A1895601, USA), and the medium was changed on day 1 . On day 3, cells were treated with the Wnt inhibitor IWP2 (72124, STEMCELL Technologies, Canada) to direct the hiPSCs into a cardiac lineage further. Starting from day 7 and after that, the cells were cultured in RPMI1640/B27 with insulin (RPMI1640/B27+ins, GIBCO, 11875093/17504044, USA), and the medium was changed every other day for the next 3 weeks. The cells were screened routinely for the presence and/or absence of lineage markers.

The differentiated hiPSC-CMs were characterized by the presence of the cardiomyocyte structural marker sarcomeric- $\alpha$-actinin, and myosin light chain 2 (MLC2V) was used as a marker for ventricular cardiomyocytes. Immunofluorescence staining was performed on day 30 . Briefly, the cells were fixed with $4 \%$ paraformaldehyde for 15 minutes, and then the cell membrane was permeabilized with $0.5 \%$ Triton X-100 for 20 minutes at room temperature. After fixation and permeabilization, the cells were blocked with $5 \%$ bovine serum albumin (BSA) in phosphate-buffered saline (PBS) at $37{ }^{\circ} \mathrm{C}$ for 30 minutes. The cells were then incubated with a rabbit anti-MLC-2V primary antibody (Proteintech, 10906-1-AP, USA, 1:200) and a mouse anti- $\alpha$-actinin (sarcomeric) primary antibody (Sigma-Aldrich, A7811, USA, 1:200) overnight at $4{ }^{\circ} \mathrm{C}$. This was followed by incubation with an Alexa Fluor-555 donkey anti-mouse IgG (AB150106, Abcam, 1:1,000) and an Alexa Fluor-488 donkey anti-rabbit IgG (AB150073, Abcam, 1:1,000) for 20-30 minutes in PBS at room temperature. After incubation, the cells were stained with 4'6-Diamidino-
2-Phenylindole (DAPI; Beyotime Biotechnology, C1002, China, 1:1,000) for 10 minutes in PBS. Immunofluorescence imaging was performed using the Leica TSC SP8 laser confocal microscopic system (Leica, DMI6000, Germany).

To further analyze the purity of the hiPSC-CMs, on day 30, cultured cells were dissociated using the human cardiomyocyte digestion solution I/II (CELLAPY, CA2011007/CA2012008, China) according to the manufacturer's instructions. Briefly, the digestion solution I was applied for 10 minutes, followed by applying the digestion solution II for 20 minutes. The Foxp3/ Transcription Factor Staining Buffer Set (Invitrogen, 005523-00, USA) was then used to fix and permeabilize the cells following the manufacturer's instructions. Briefly, cells were incubated with $200 \mu \mathrm{L}$ fixation/permeabilization solution for 30 minutes on ice. The permeabilized cells were then incubated with a FITC-conjugated anti-cardiac troponin T antibody (1C11-FITC; Abcam, ab105439, UK, 1:100). The stained cardiomyocytes were analyzed using a flow cytometer (FACS Canto, BD, USA). Histogram plots were generated using the FlowJo software (V10.0.7, $\mathrm{BD}, \mathrm{USA})$ to quantify the percentage of live cells that were cardiac troponin $\mathrm{T}$ (CTNT) positive.

\section{Electrophysiological recordings}

The cultured hiPSC-CMs $\left(4 \times 10^{4}\right.$ to $8 \times 10^{4}$ cells $/ 35 \mathrm{~mm}$ dish) were first digested with $1 \mathrm{~mL} 1 / 4 \mathrm{X}$ Accutase (7920, STEMCELL Technologies, Canada, diluted in PBS) for one minute. The cells were then washed once with baseline extracellular fluid and transferred to the stage of an inverted microscope (ECLIPSE Ti-U, Nikon, Japan) for patch-clamp recording. The hiPSC-CMs were continuously perfused with an extracellular solution through a "Y-tube" system with a solution exchange time of 1 minute. Drugs were applied through the "Y-tube" system for 3 minutes each time. Whole-cell patch-clamp recordings on the hiPSC-CMs were performed using Axopatch 700B (Axon Instruments, Inc., Union City, CA, USA) amplifiers under an inverted microscope at room temperature $\left(22-25^{\circ} \mathrm{C}\right)$. Patch pipettes were fabricated from $1.5 \mathrm{~mm}$ outside diameter thin-walled borosilicate glass tubing (Sutter Instruments Co, Novato, CA, USA) using the Flaming/Brown micropipette puller P97 (Sutter Instruments Co, Novato, CA, USA). The patch pipette tips' final resistance was 2-4 M $\Omega$ after heat polish and internal solution filling. After "gigaseal" between the patch pipette and cell membranes was formed, gentle suction 
was used to rupture the cell membrane and to establish a whole-cell configuration. All current signals were digitized with a sampling rate of $10 \mathrm{kHz}$ and filtered at a cutoff frequency of $2 \mathrm{kHz}$ (Digidata 1550A, Axon Instruments, Inc., Union City, CA, USA). The spontaneous action potentials were recorded by the gap-free mode with a sampling rate of $1 \mathrm{kHz}$ and filtered at a cutoff frequency of $0.5 \mathrm{kHz}$. Series resistance was compensated by $40-80 \%$. If the series resistance was more than $10 \mathrm{M} \Omega$ or changed significantly during the experiments, the recordings were discarded from further analysis. For the $I_{\mathrm{Na}}$ recordings, the linear leakage currents were subtracted by using an on-line "p-5" procedure (in which the currents evoked by 5 hyperpolarizing pulses with one-fifth amplitude of the test pulse were summed and added to the current trace of interest). For the $I_{\mathrm{Ca}}$ recordings, $1.0 \mu \mathrm{M}$ tetrodotoxin (TTX) was added to the extracellular solution to block the $I_{\mathrm{Na}}$.

\section{Solutions and drugs}

The pipette internal solution for action potential recording contained the following in $\mathrm{mM}$ concentrations: $\mathrm{KCl} 150$, $\mathrm{NaCl} 5, \mathrm{CaCl}_{2}$ 2, EGTA 5, HEPES 10, and MgATP 5 (pH $7.2, \mathrm{KOH})$. For $I_{\mathrm{Na}}$ and $I_{\mathrm{Ca}}$ recordings, the pipette internal solution contained the following in $\mathrm{mM}$ concentrations: CsCl 130, $\mathrm{NaCl} 5$, EGTA 10, HEPES 10, and Mg-ATP $5(\mathrm{pH} 7.2, \mathrm{CsOH})$. For $I_{\mathrm{f}}$ recordings, the pipette internal solution contained the following in $\mathrm{mM}$ concentrations: $\mathrm{NaCl} 6$, K-aspartate $130, \mathrm{MgCl}_{2} 2, \mathrm{CaCl}_{2}$ 2, EGTA 5, $\mathrm{Na}_{2}$ ATP 2, NaGTP 0.1, cAMP 0.2, and HEPES 5 (pH $7.2, \mathrm{KOH})$. For $I_{\mathrm{k} 1}$ recordings, the pipette internal solution contained the following in $\mathrm{mM}$ concentrations: $\mathrm{KCl} 140$, $\mathrm{MgCl}_{2}$ 1, glucose 10, MgATP 2, EGTA 5, and HEPES $5(\mathrm{pH} 7.2, \mathrm{KOH})$. For $I_{\mathrm{kr}}$ recordings, the pipette internal solution contained the following in $\mathrm{mM}$ concentrations: CsCl 140, $\mathrm{MgCl}_{2}$ 2, glucose 10, and HEPES 10 (pH 7.4, $\mathrm{CsOH})$.

Both the baseline extracellular solution and the extracellular solution for action potential recording contained the following in $\mathrm{mM}$ concentrations: $\mathrm{NaCl}$ 140, $\mathrm{KCl} 5, \mathrm{CaCl}_{2} 1, \mathrm{MgCl}_{2}$ 1, glucose 10, and HEPES $10(\mathrm{pH} 7.4, \mathrm{NaOH})$. For $I_{\mathrm{Na}}$ recordings, the extracellular solution contained the following in $\mathrm{mM}$ concentrations: choline chloride $100, \mathrm{NaCl} 50, \mathrm{MgCl}_{2} 2$, 4-AP 5, HEPES 10, glucose 10, $\mathrm{CsCl} 20$, and $\mathrm{CdCl}_{2} 0.3(\mathrm{pH} 7.35, \mathrm{HCl}$ ). For $I_{\mathrm{Ca}}$ recordings, the extracellular solution contained the following in $\mathrm{mM}$ concentrations: $\mathrm{NaCl} 135, \mathrm{KCl} 5.4, \mathrm{CaCl}_{2}$ $1.8, \mathrm{MgCl}_{2}$ 1.0, $\mathrm{NaH}_{2} \mathrm{PO}_{4}$ 0.33, HEPES 10 , and glucose
$10(\mathrm{pH} 7.35, \mathrm{NaOH})$. For $I_{\mathrm{f}}$ recordings, the extracellular solution contained the following in $\mathrm{mM}$ concentrations: $\mathrm{NaCl}$ 137.7, $\mathrm{KCl} 5.4, \mathrm{MgCl}_{2}$ 1, $\mathrm{CaCl}_{2}$ 1.8, HEPES 5, $\mathrm{NaOH} 2.3$, glucose 10, 4-aminopyridine 2, $\mathrm{BaCl}_{2} 5$, and $\mathrm{CdCl}_{2} 0.2(\mathrm{pH} 7.4, \mathrm{NaOH})$. For $I_{\mathrm{K} 1}$ recordings, the extracellular solution contained the following in $\mathrm{mM}$ concentrations : $\mathrm{NaCl} 136, \mathrm{KCl} 5.4, \mathrm{MgCl}_{2} 1, \mathrm{NaH}_{2} \mathrm{PO} 4$ 0.33 , glucose 10, HEPES 10, TTX 0.01, and 4-AP 4 (pH, $7.35, \mathrm{NaOH})$. For $I_{\mathrm{Kr}}$ recordings, the extracellular solution contained the following in $\mathrm{mM}$ concentrations: $\mathrm{CsCl} 140$, $\mathrm{MgCl}_{2}$ 2, EGTA 10, and HEPES 10 (pH 7.2, CsOH).

DMED was purchased from Waterstone Technology (Carmel, IN, USA). Yohimbine, idazoxan, and phentolamine were purchased from Sigma-Aldrich (St Louis, USA). In this study, $1.0 \mu \mathrm{M}$ yohimbine, $10 \mu \mathrm{M}$ idazoxan (19), and $1.0 \mu \mathrm{M}$ phentolamine (20) were used. All other chemical reagents were of analytical grade and purchased from Sinopharm Chemical Reagent Co., Ltd (Shanghai, China).

\section{Data analysis}

Data were analyzed using Clampfit 10.5 and Origin 8.0 (OriginLab, Northampton, MA, USA). For the construction of the concentration-response curve, the data were fitted with the equation $y=\mathrm{A} 1+(\mathrm{A} 2-\mathrm{A} 1) /[1$ $+10^{\left.\left.\wedge(\text { LOG } x 0-x)^{*} p\right)\right)}$, in which $X$ is the drug concentration, $X 0$ is the drug concentration required to produce $50 \%$ inhibition of the spontaneous action potential frequency, A1 denotes the initial value, A2 denotes the final value, and $p$ denotes the power. For the construction of the $\mathrm{Na}^{+}$ channel activation curves, the normalized conductance was plotted against the test pulse potentials. It was fitted to a Boltzmann function according to the following equation: $G_{\mathrm{Na}} /_{\max } G_{\mathrm{Na}}=1 /\left[1+\exp \left(V_{\mathrm{g} 0.5}-V_{\mathrm{g}}\right) / k_{\mathrm{g}}\right]$, where ${ }_{\text {max }} G_{\mathrm{Na}}$ is the maximal value for $G_{\mathrm{Na}}, V_{\mathrm{g} 0.5}$ is the potential at which $G_{\mathrm{Na}}$ is half of ${ }_{\max } G_{\mathrm{Na}}$, and $k_{\mathrm{g}}$ is the slope factor. The $\mathrm{Na}^{+}$ conductance $\left(G_{\mathrm{Na}}\right)$ of the membrane was calculated by using the following equation: $G_{\mathrm{Na}}=I_{\mathrm{Na}_{\mathrm{a}}} /\left(V_{\mathrm{g}}-V_{\mathrm{r}}\right)$, where $I_{\mathrm{Na}}$ is the peak amplitude of the $I_{\mathrm{Na}}, V_{\mathrm{g}}$ is the membrane potential achieved using a step pulse, and $V_{\mathrm{r}}$ is the reversal potential for $\mathrm{Na}^{+}$channels. For the construction of $\mathrm{Na}^{+}$ channel inactivation curves, the normalized $I_{\mathrm{Na}}$ was plotted against the conditioning pulse potentials. It was fitted to a Boltmann function according to the following equation: I/ $I_{\max }=1 /\left[1+\exp \left(V_{1 / 2}-V\right) / k\right]$, in which $V_{1 / 2}$ is the membrane potential at which half-maximal channel is inactivated and $k$ is the slope factor. The construction of the $\mathrm{Ca}^{2+}$ channel 


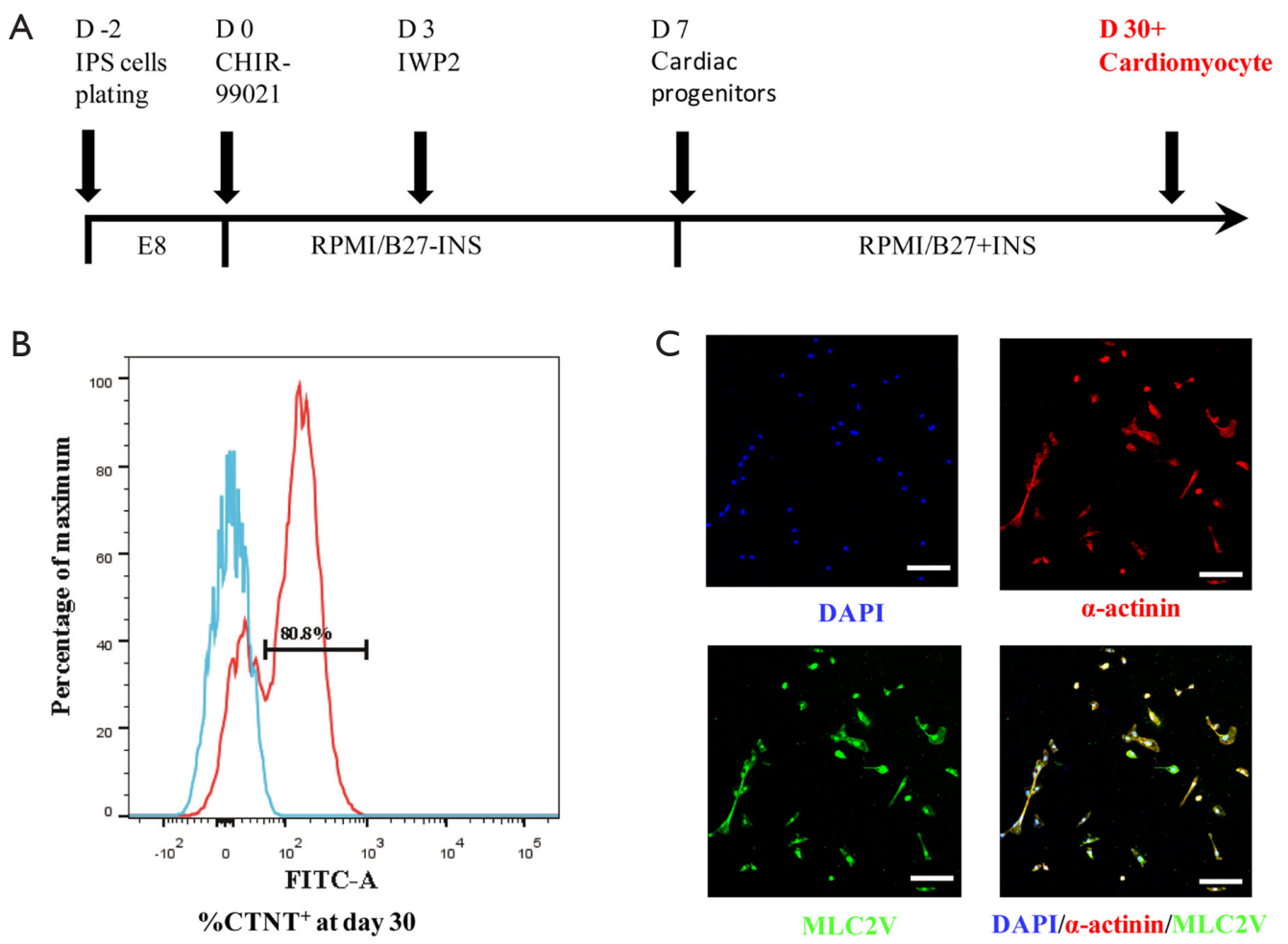

Figure 1 The generation and identification of hiPSC-derived cardiomyocytes. (A) A schematic diagram of the differentiation process from hiPSC into cardiomyocyte. (B) The percentage of CTNT positive cardiomyocytes measured by flow cytometry on day 30 , n=3 independent biological replicates. (C) Immunofluorescence staining for $\alpha$-actinin (a cardiomyocyte structural markers), and MLC2V (a marker for ventricular cardiomyocytes). Scale bar, $100 \mu \mathrm{m}$. hiPSC, human-induced pluripotent stem cell; CTNT, cardiac troponin T; MLC2V, myosin light chain 2 .

activation and inactivation curves were similar to that for the $\mathrm{Na}^{+}$channel. The time constant of activation and inactivation was fitted to a single-exponential function [I $\left.=A_{0}+A_{1} \exp (-t / \tau)\right]$, where $A_{0}$ denotes the initial value, $A_{1}$ denotes the peak value, and $\tau$ denotes the time constant.

\section{Statistical analysis}

Data values are expressed as mean \pm SEM with sample sizes ( $n$, the number of recorded cardiomyocytes). Each experiment was repeated 5-6 times in different cardiomyocytes. Each cardiomyocyte was used only one time. Statistical analysis was performed using Origin 8.0 software. One-way analysis of variance (ANOVA) was used for multiple comparisons, and paired and/or unpaired twotailed Student's $t$-test was used for evaluating the statistical significance of differences between two group means. A P value $<0.05$ was considered statistically significant.

\section{Results}

\section{The generation and identification of hiPSC-CMs}

The hiPSCs were differentiated into beating cardiomyocytes according to the schematic process shown in Figure $1 \mathrm{~A}$. Flow cytometry analysis revealed that $80.3 \% \pm 0.42 \%$ $(n=3)$ of cells differentiated from the hiPSCs were CTNT positive on day 30 of differentiation (Figure 1B). This was further confirmed by immunofluorescence staining, which demonstrated the cardiomyocyte structural marker sarcomeric- $\alpha$-actinin and the ventricular cardiomyocyte marker MLC2V the differentiated cells on day 30 . The majority of the hiPSC-CMs were ventricular cardiomyocytes (Figure 1C).

The patch-clamp recordings of the spontaneously beating cardiomyocytes demonstrated that the action potentials were all ventricular-like shapes (Figure $2 A$ ). This is in agreement with the classification criteria of hiPSC- 
A

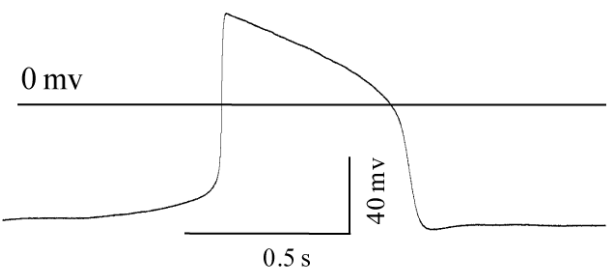

C

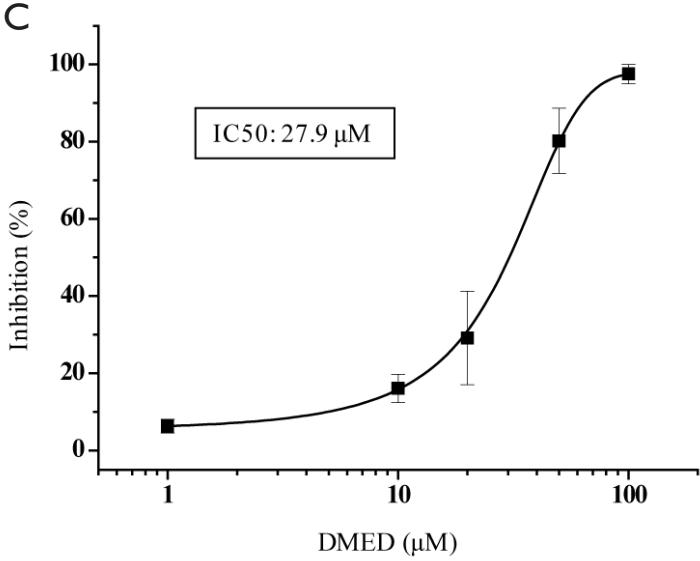

B

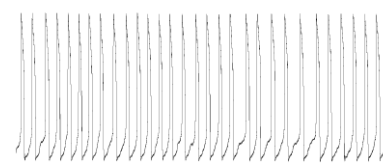

Control

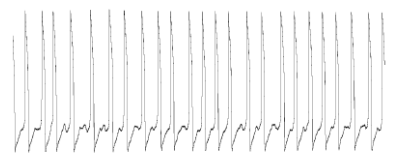

$10 \mu \mathrm{M}$ DMED

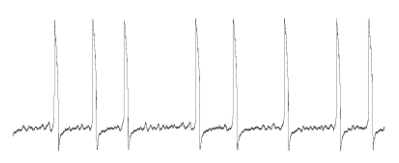

$50 \mu \mathrm{M}$ DMED

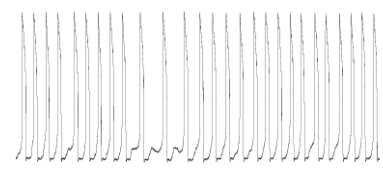

Recovery

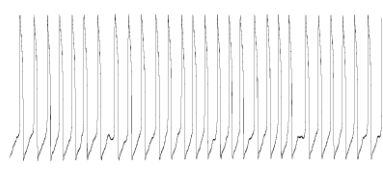

1.0 $\mu \mathrm{M}$ DMED

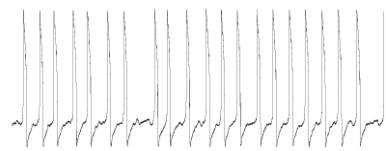

$20 \mu \mathrm{M}$ DMED
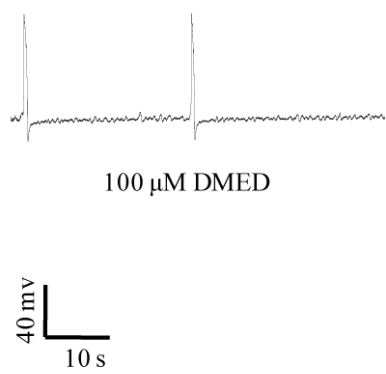

Figure 2 The dose-dependent inhibition of action potential frequency of ventricular-like hiPSC-CMs by DMED. (A) A representative spontaneous action potential trace. (B) A 1-minute recording of a typical spontaneous action potential trace after treatment with different concentrations of DMED. (C) The inhibition of spontaneous action potential frequency by DMED at various concentrations. Abscissa: concentrations of DMED. Ordinate: \% inhibition of the peak frequency of the spontaneous action potential in 1 minute. Each point represents the mean \pm SEM ( $\mathrm{n}=6)$. hiPSC-CMs, human-induced pluripotent stem cell-derived cardiomyocytes; DMED, dexmedetomidine; SEM, standard error of the mean.

CMs reported by Ma and colleagues (21).

\section{The effects of DMED on the frequency of spontaneous action potentials of ventricular-like biPSC-CMs}

The typical spontaneous action potential traces were inhibited by the $\alpha_{2}$-adrenoceptor agonist DMED. At concentrations of $1.0,10,20,50$, and $100 \mu \mathrm{M}$, DMED inhibited the frequency of action potentials shown by hiPSC-CMs by $6.3 \% \pm 1.8 \%, 16.1 \% \pm 3.7 \%, 29.1 \% \pm 12.1 \%, 80.2 \% \pm 8.4 \%$, and $97.5 \% \pm 2.5 \%$, respectively (Figure $2 B$ ). Further analysis demonstrated that DMED dose-dependently inhibited the frequency of spontaneous action potentials of hiPSC-CMs, with an $\mathrm{IC}_{50}$ value of $27.9 \mu \mathrm{M}$ (Figure $2 C$ ). Therefore, $30 \mu \mathrm{M}$ DMED was used for the duration of our studies.

\section{The effects of DMED on the action potential parameters of ventricular-like biPSC-CMs}

To explore the mechanisms by which DMED decreased the frequency of spontaneous action potentials in hiPSC$\mathrm{CMs}$, we examined the resting potential, threshold potential, amplitude, and maximal diastolic potential (Figure $3 A$ ). DMED did not significantly affect the resting potential $(-49.8 \pm 2.0$ vs. $-46.1 \pm 2.2 \mathrm{mV})$, threshold potential $(-41.7 \pm 1.5$ vs. $38.7 \pm 1.8 \mathrm{mV})$, amplitude $(102.0 \pm 4.7$ vs. $96.5 \pm 4.8 \mathrm{mV})$, or maximal diastolic potential $(-67.4 \pm 2.4 v s .63 .3 \pm 2.4 \mathrm{mV})$ of the action potentials recorded from hiPSC-CMs (Figure 3B,C,D,E). However, DMED significantly prolonged the action potential duration at $90 \%$ repolarization $\left(\mathrm{APD}_{90}\right.$; $461.0 \pm 20.9$ vs. $561.0 \pm 16.8$ ms; Figure 3F). 
A

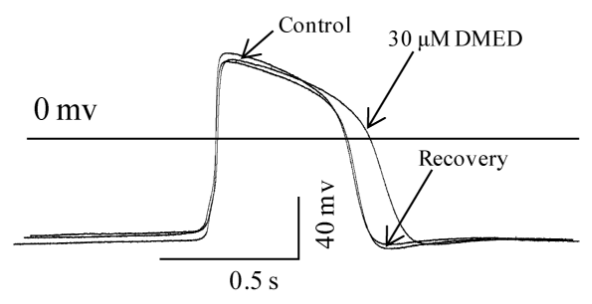

$\mathrm{D}$

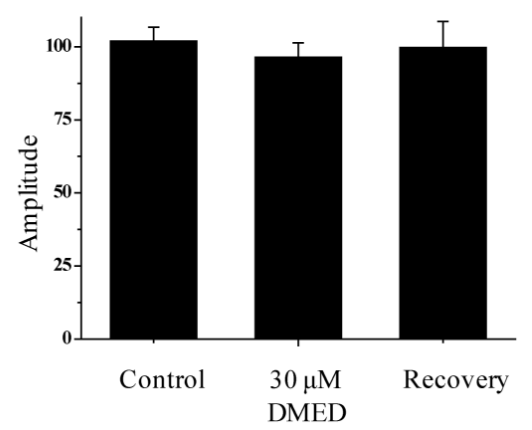

B

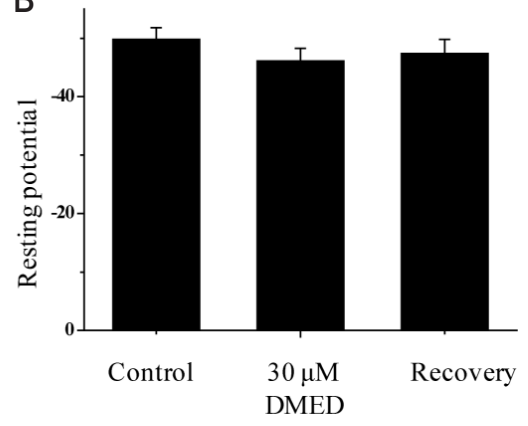

$\mathrm{E}$

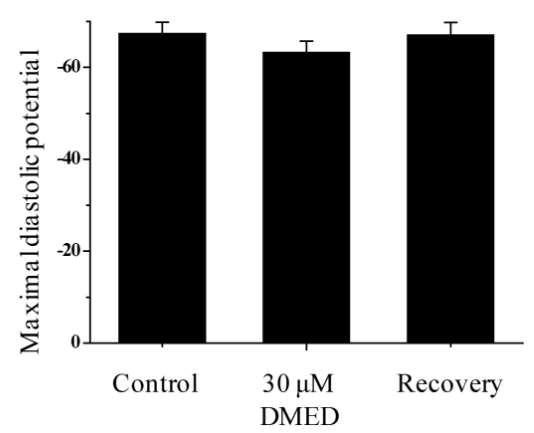

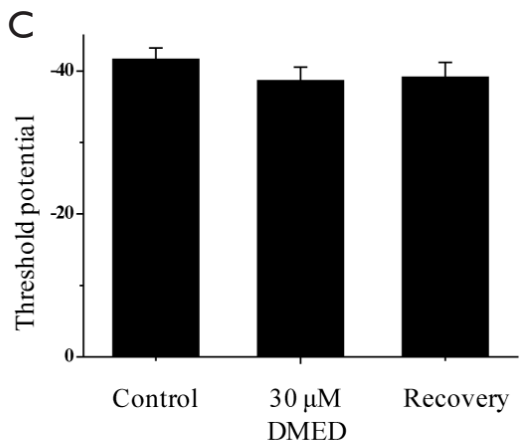

$\mathrm{F}$

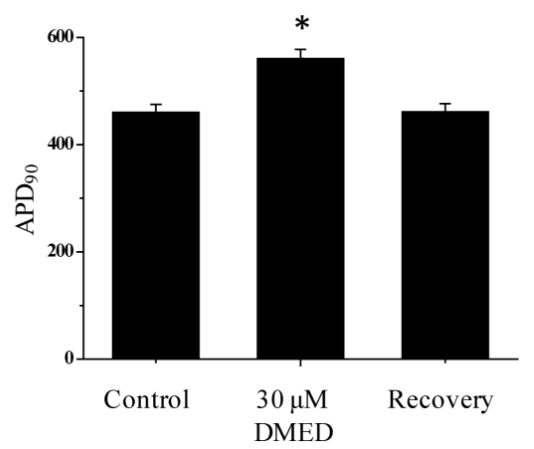

Figure 3 The effects of DMED on the action potential parameters of ventricular-like hiPSC-CMs (resting potential, threshold potential, amplitude, maximal diastolic potential, and $\mathrm{APD}_{9_{0}}$ ). (A) A representative recording of the action potential with or without DMED. (B,C,D,E,F) The action potential parameters with or without DMED. *, $\mathrm{P}<0.05$ compared to vehicle control (n=5). hiPSC-CMs, humaninduced pluripotent stem cell-derived cardiomyocytes; $\mathrm{DMED}$, dexmedetomidine; $\mathrm{APD}_{90}$, action potential duration at $90 \%$ repolarization.

\section{The effects of DMED on the hyperpolarization-activated pacemaker currents of hiPSC-CMs}

Pacemaker currents $\left(I_{\mathrm{f}}\right)$ play a very important role in determining and maintaining the spontaneous rhythm of action potentials in cardiac sinoatrial node cells $(22,23)$; therefore, the effects of DMED on $I_{\mathrm{f}}$ were examined. DMED did not affect the shape or amplitudes of the current/voltage (I/V) relationship curve of $I_{\mathrm{f}}$ for both the steady-state and the peak tail currents (Figure 4).

\section{The effects of DMED on the $K^{+}$channel currents of biPSC-CMs}

Potassium ion channel currents $\left(I_{\mathrm{k}}\right)$ play a crucial role in determining and maintaining the plateau stage of action potentials in cardiac ventricular cells. As such, the effects of DMED on $I_{\mathrm{k}}$ were investigated. DMED did not affect the shape or the amplitudes of the I/V/l relationship curves of
$I_{\mathrm{k} 1}$ and $I_{\mathrm{kr}}$ (Figure 5).

\section{The effects of DMED on the $\mathrm{Na}^{+}$channel currents of biPSC-CMs}

Sodium ion channels determine cardiac excitability and electrical conduction velocity. Blocking the sodium channel currents $\left(I_{\mathrm{Na}}\right)$ or slowing the action potential upstroke would suppress supraventricular and ventricular arrhythmias in the clinical setting (24). DMED inhibited the amplitude of the $I_{\mathrm{Na}}$, but did not affect the shape of the I/V relationship curves of the $\mathrm{Na}^{+}$channel (Figure 6A). The representative recordings of the $I_{\mathrm{Na}}$ in the absence or presence of DMED are shown in Figure 6B. DMED neither affected the $\mathrm{Na}^{+}$ channel steady-state activation curves nor the steady-state inactivation curves (Figure 6C,D). However, it significantly decreased the time constant of activation at potentials from -40 to $-20 \mathrm{mv}$ (Figure $6 \mathrm{E}$ ), and significantly increased the time constant of inactivation (Figure $6 F$ ). 


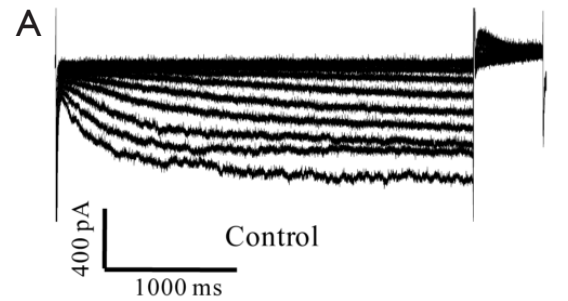

B

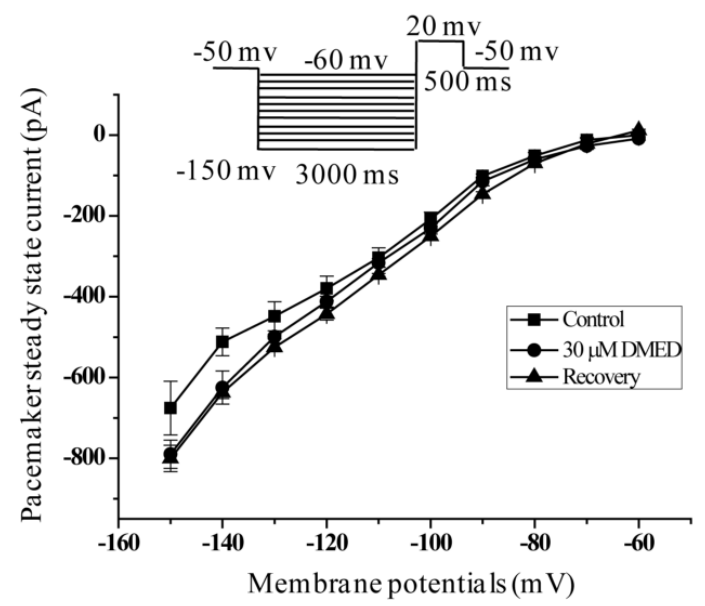

C

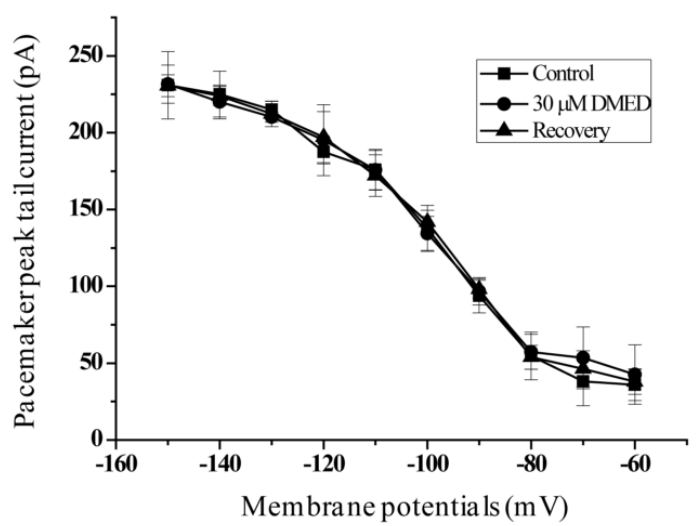

Figure 4 The effects of DMED on hyperpolarization-activated pacemaker currents of hiPSC-CMs. (A) A representative recording of pacemaker current traces with or without DMED. (B) The I/V curves of pacemaker steady state currents with or without DMED. (C) The I/V curves of pacemaker peak tail currents with or without DMED. Each point represents the mean \pm SEM $(n=6)$. hiPSC-CMs, humaninduced pluripotent stem cell-derived cardiomyocytes; DMED, dexmedetomidine; I/V, current/voltage; SEM, standard error of the mean.

\section{The effects of DMED on the $\mathrm{L}-\mathrm{Ca} \mathrm{a}^{2+}$ channel currents of biPSC-CMs}

In our study, DMED inhibited the L type calcium ion channel current (ICa) amplitude, but it did not affect the shape of the I/V relationship curves of the $\mathrm{Ca}^{2+}$ channel (Figure $7 A$ ). Figure $7 B$ illustrates the representative recordings of the $I_{\mathrm{Ca}}$ in the absence or presence of DMED. DMED did not affect the steady-state activation curves of the $\mathrm{Ca}^{2+}$ channel (Figure 7C), but it significantly decreased the time constant of activation at potential $-20 \mathrm{mv}$ (Figure $7 D$ ). Also, DMED did not affect the steady-state inactivation curves of the $\mathrm{Ca}^{2+}$ channel either (Figure $7 E$ ), but instead significantly increased the time constant of inactivation (Figure $7 F$ ).

\section{The DMED-mediated inbibition of the frequency of spontaneous action potential, $I_{N a}$, and $I_{C a}$ in hiPSC-CMs was independent of the $\alpha_{2}$-adrenoceptor, imidazoline receptor, and the $\alpha_{1}$-adrenoceptor}

DMED is a well-known, highly selective, and potent $\alpha_{2}$ adrenoceptor agonist (14). We examined whether the $\alpha_{2}$ adrenoceptor might be responsible for mediating the inhibitory effects of DMED on hiPSC-CMs. A dose of 10 $\mu M$ yohimbine (an $\alpha 2$-adrenoceptor antagonist) affected the shape of the action potentials, whereas a dose of $1 \mu \mathrm{M}$ did not, and therefore, $1 \mu \mathrm{M}$ yohimbine was used in this study. Yohimbine could not antagonize the inhibitory effects of DMED on the frequency of spontaneous action potentials or the peak values of the $I_{\mathrm{Na}}$ and $I_{\mathrm{Ca}}$ in hiPSC- 
A

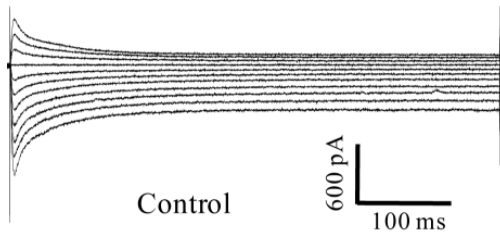

B

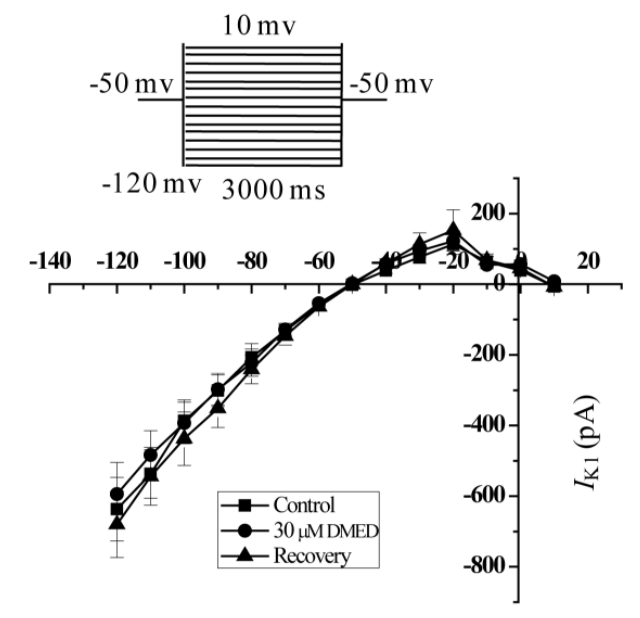

Membrane potentials (mV)

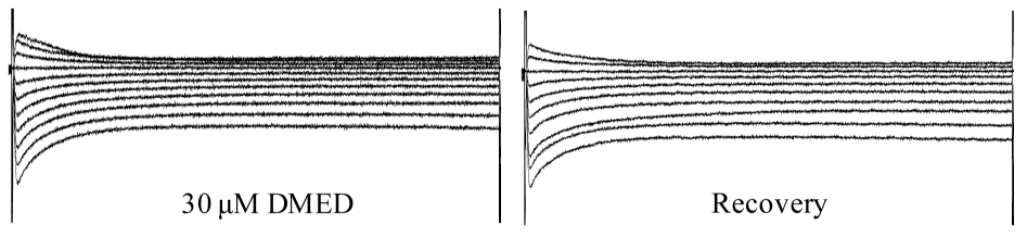

C

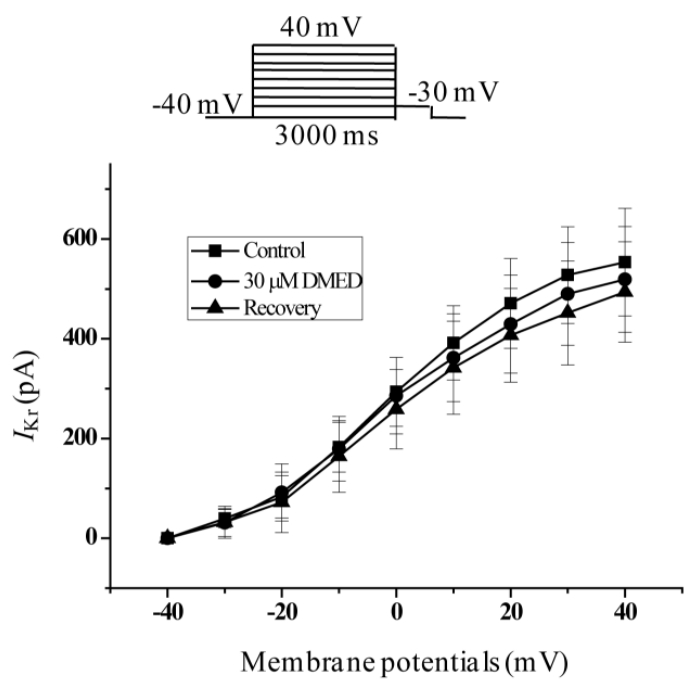

Figure 5 The effects of DMED on the potassium channel currents of hiPSC-CMs. (A) A representative recording of the $I_{\mathrm{K} 1}$ traces with or without DMED. (B) The I/V curves of $I_{\mathrm{K} 1}$ with or without DMED. (C) The I/V curves of $I_{\mathrm{Kr}}$ with or without DMED. Each point represents the mean \pm SEM $(n=5)$. hiPSC-CMs, human-induced pluripotent stem cell-derived cardiomyocytes; DMED, dexmedetomidine; I/ V, current/voltage; SEM, standard error of the mean.

CMs. DMED in the presence of yohimbine reduced the frequency of spontaneous action potentials by $55.2 \% \pm 6.2 \%$, which was comparable to the inhibitory effects produced by DMED alone with a reduction of $52.3 \% \pm 5.1 \%$ (Figure $8 A$ ). DMED in the presence of yohimbine inhibited the peak values of the $I_{\mathrm{Na}}$ and $I_{\mathrm{Ca}}$ by $45.1 \% \pm 5.2 \%$ and $65.1 \% \pm 4.2 \%$, respectively, which was again similar to the inhibitory effects produced by DMED alone with reductions of $45.3 \% \pm 7.1 \%$ and $63.6 \% \pm 6.1 \%$, respectively (Figure $8 B, C$ ).

DMED is not only an $\alpha_{2}$-adrenoceptor agonist, but it is also an imidazole derivative that acts as an imidazole receptor agonist. We investigated whether the imidazole receptor could be responsible for mediating the inhibitory effects of DMED on the frequency of spontaneous action potential and the peak values of the $I_{\mathrm{Na}}$ and $I_{\mathrm{Ca}}$ in hiPSC-CMs. Idazoxan, which is both an $\alpha_{2}$-adrenoceptor antagonist and an imidazole receptor antagonist, did not antagonize the inhibitory effects of DMED on the frequency of spontaneous action potentials $(59.2 \% \pm 5.2 \%$ vs. $52.3 \% \pm 5.1 \%)$, nor did it affect the peak values of the $I_{\mathrm{Na}}$ $(46.1 \% \pm 6.2 \%$ vs. $45.3 \% \pm 7.1 \%)$ and $I_{\mathrm{Ca}}(64.1 \% \pm 5.4 \%$ vs. $63.6 \% \pm 6.1 \%$ ) in hiPSC-CMs (Figure 9).

In addition to the activation of the $\alpha_{2}$-adrenoceptor and the imidazole receptor, DMED can also activate the $\alpha_{1}$ adrenoceptor, although the binding capability is relatively low compared with that of the $\alpha_{2}$-adrenoceptor. We further examined whether the $\alpha_{1}$-adrenoceptor mediated the inhibitory effects of $30 \mu M$ DMED on the frequency of spontaneous action potential and the peak values of the $I_{\mathrm{Na}}$ and $I_{\mathrm{Ca}}$ in hiPSC-CMs. The $\alpha_{1}$-adrenoceptor antagonist phentolamine did not antagonize the inhibitory effects of DMED on the frequency of action potentials $(57.3 \% \pm 7.2 \%$ vs. $52.3 \% \pm 5.1 \%)$, nor the peak values of the $I_{\mathrm{Na}}(47.6 \% \pm 5.8 \%$ vs. $45.3 \% \pm 7.1 \%)$ and $I_{\mathrm{Ca}}(66.7 \% \pm 6.4 \%$ vs. 
A
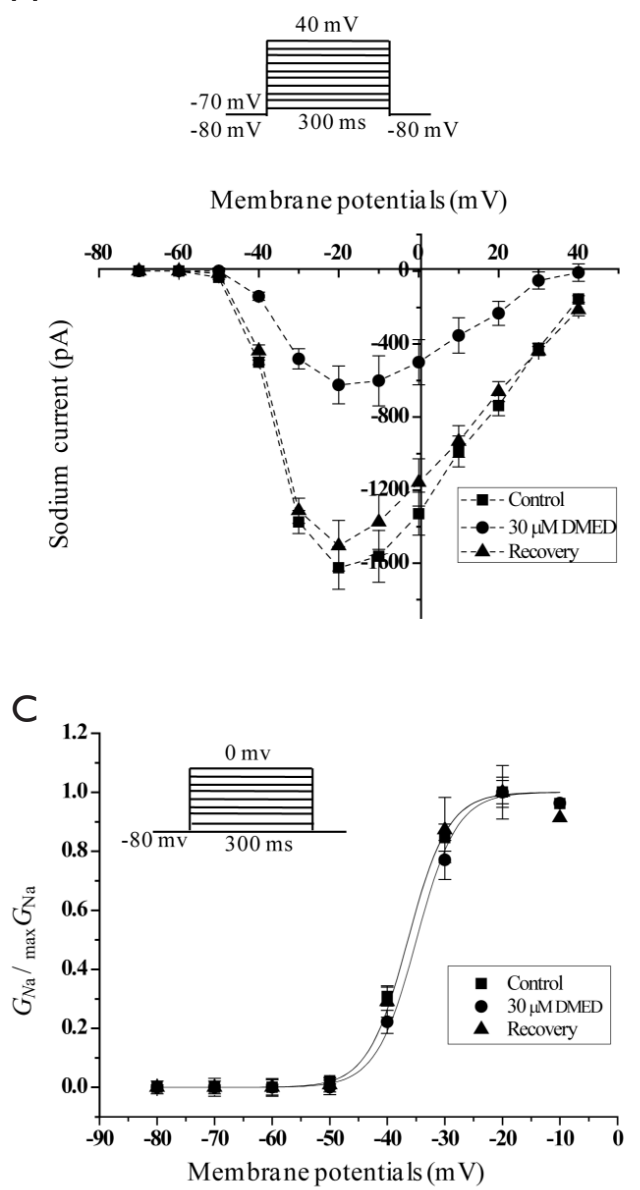

E

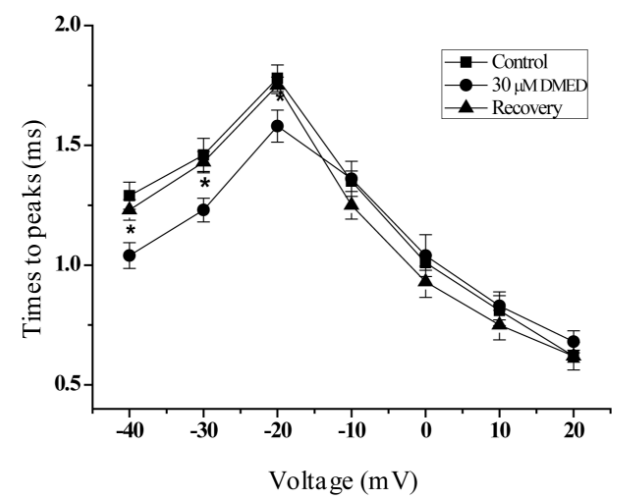

B
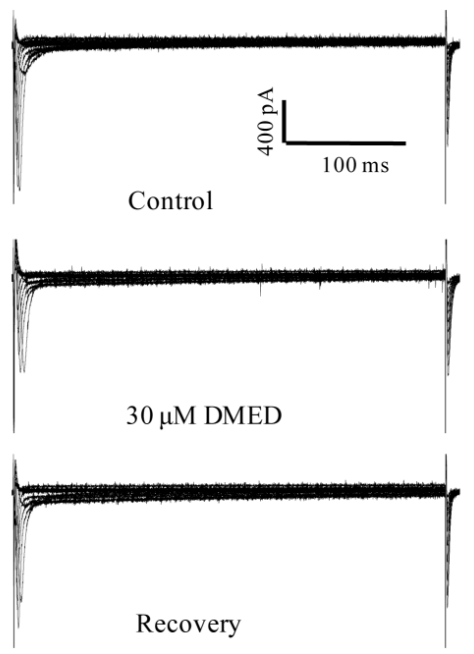

D

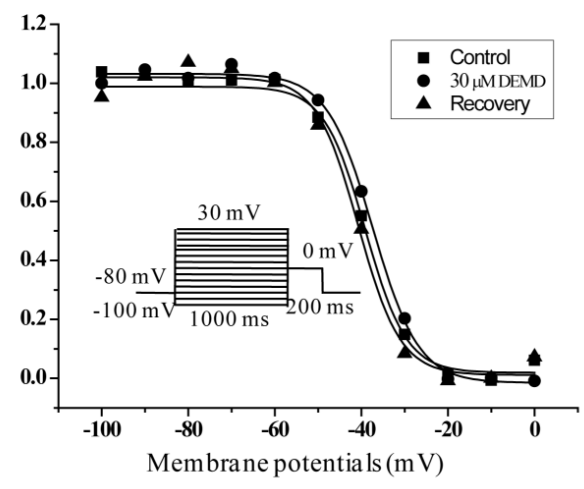

$\mathrm{F}$

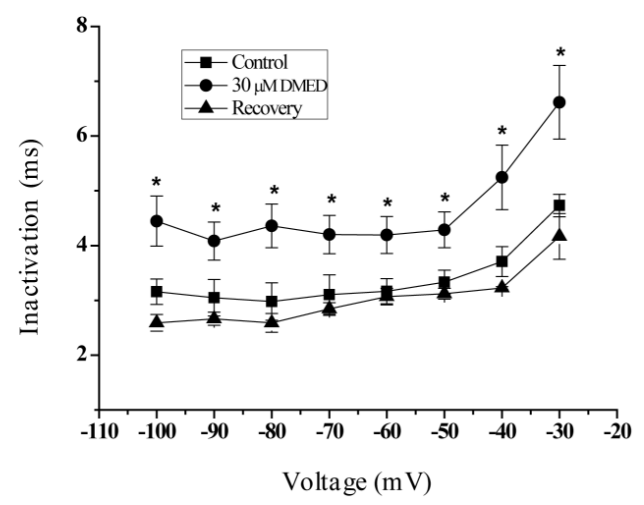

Figure 6 Inhibition of the hiPSC-CMs $\mathrm{Na}^{+}$channels by DMED. (A) The I/V relationship curves of the $\mathrm{Na}^{+}$channels in the absence or presence of DMED. (B) A representative recording in the absence or presence of DMED. (C) The $\mathrm{Na}^{+}$channel activation curves in the absence or presence of DMED. (D) The effects of DMED on the steady-state inactivation of $\mathrm{Na}^{+}$channels. $(\mathrm{E}, \mathrm{F})$ Data for the development of activation and inactivation were both fitted to a single-exponential function and plotted as a function of the development pulse voltage. The average time to reach the peaks and inactivation are shown. Each point represents the mean \pm SEM. * $\mathrm{P}<0.05$ compared to the vehicle control $(\mathrm{n}=6)$. hiPSC-CMs, human-induced pluripotent stem cell-derived cardiomyocytes; DMED, dexmedetomidine; SEM, standard error of the mean. 
A

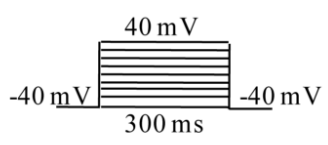

Membrane potentials $(\mathrm{mV})$

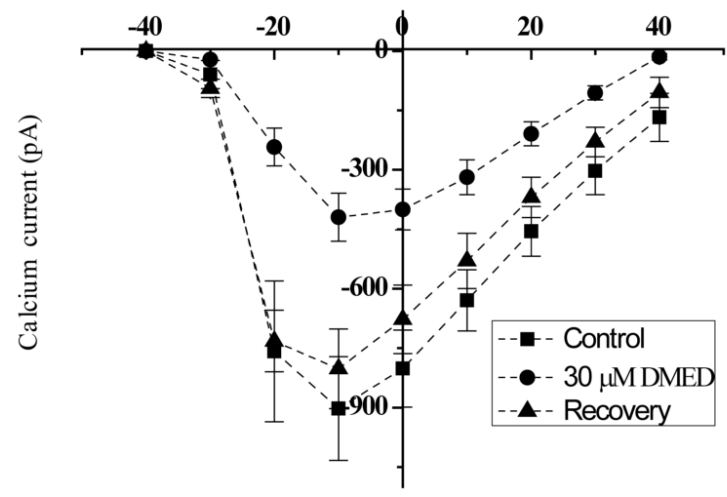

C

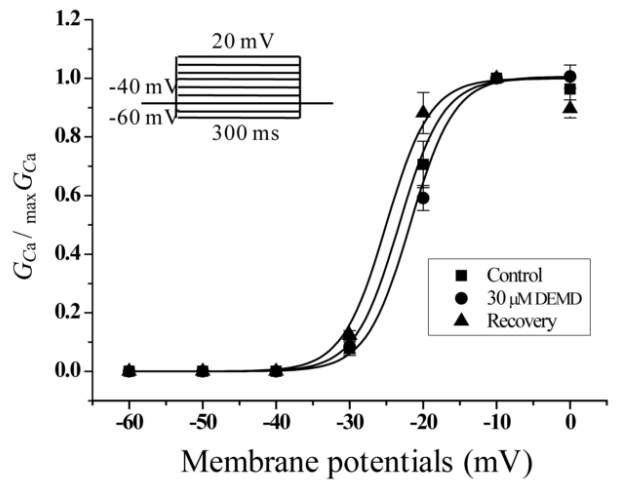

$E$

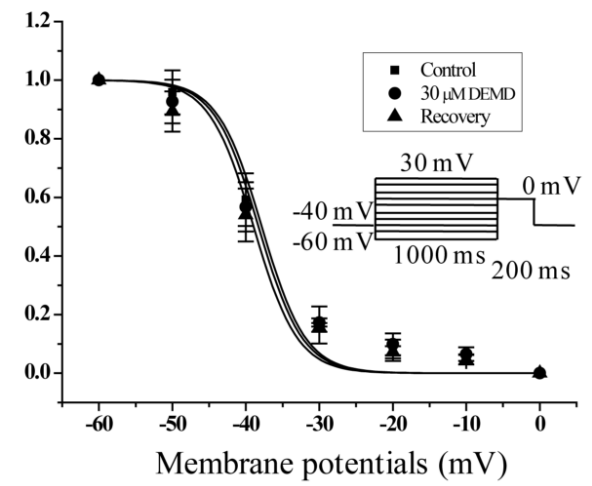

B
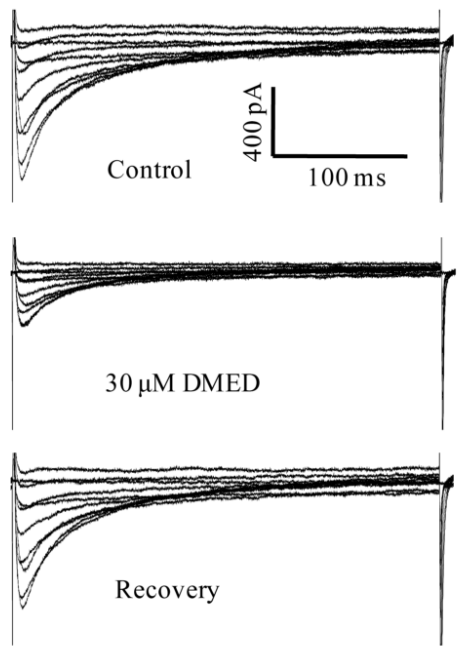

D

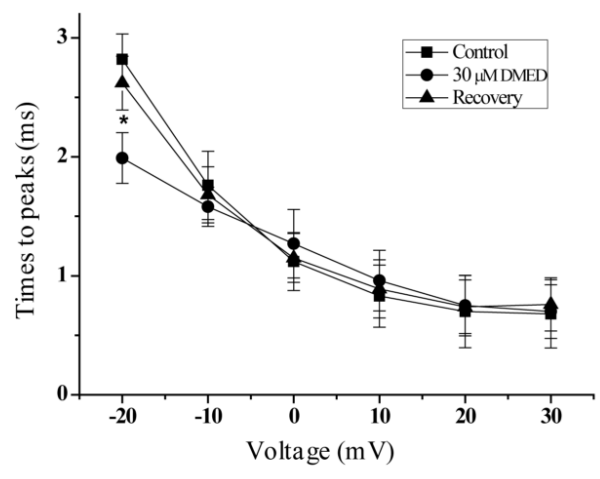

$\mathrm{F}$

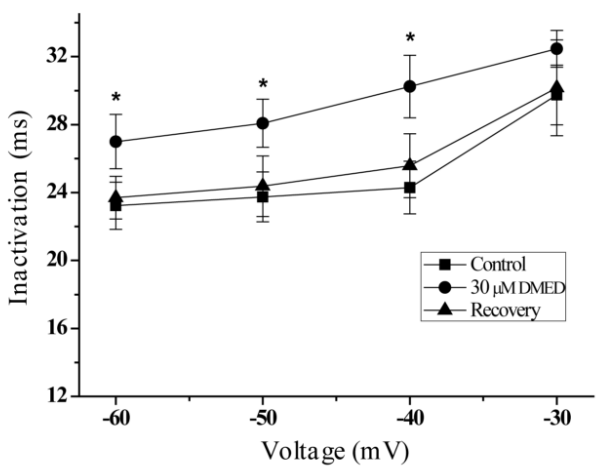

Figure 7 The inhibition of hiPSC-CMs $\mathrm{Ca}^{2+}$ channels by DMED. (A) The I/V relationship curves of the $\mathrm{Ca}^{2+}$ channels with or without DMED. (B) A typical recording with or without DMED. (C) The $\mathrm{Ca}^{2+}$ channel activation curves with or without DMED. (D) The time constant of activation for hiPSC-CM Ca ${ }^{2+}$ channels. (E) The effects of DMED on the steady-state inactivation of the $\mathrm{Ca}^{2+}$ channels. (F) The time constant of inactivation for hiPSC-CM Ca ${ }^{2+}$ channels. Each point represents the mean $\pm \mathrm{SEM}$. ${ }^{*} \mathrm{P}<0.05$ compared to the vehicle control ( $\mathrm{n}=5$ ). hiPSC-CMs, human-induced pluripotent stem cell-derived cardiomyocytes; DMED, dexmedetomidine; I/V, current/voltage; SEM, standard error of the mean. 
A
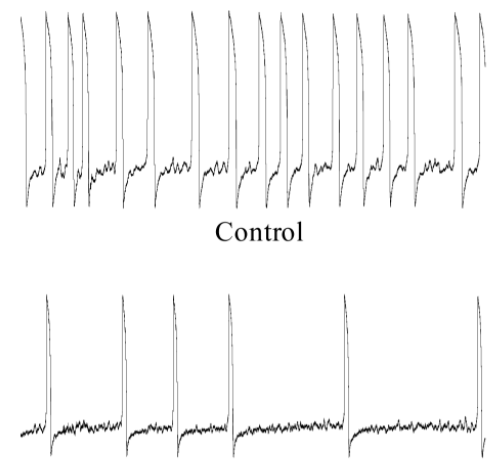

$1.0 \mu \mathrm{M}$ Yohimbine $+30 \mu \mathrm{M}$ DMED

B

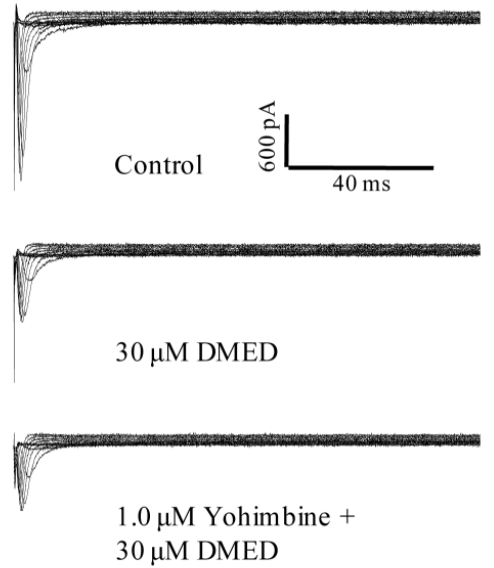

Recovery
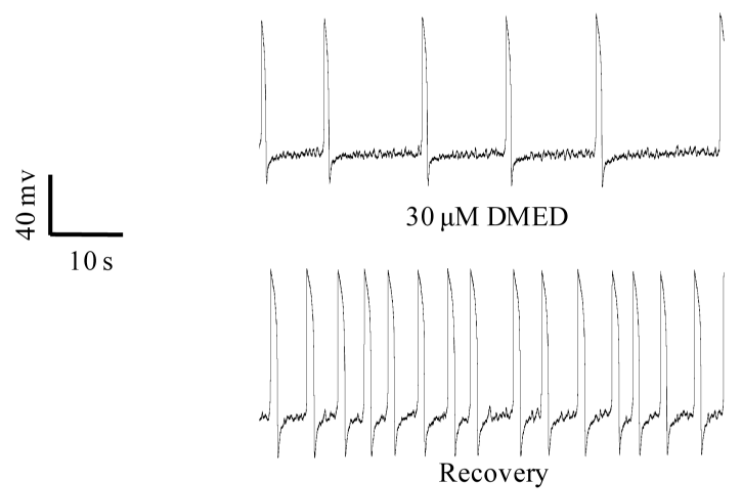

C

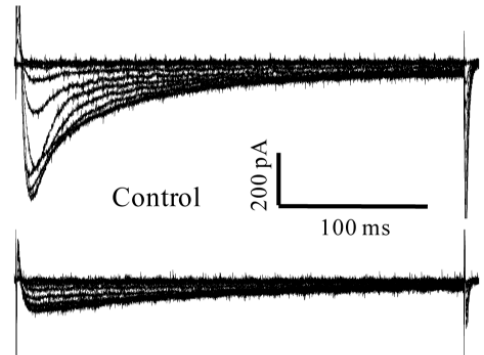

$30 \mu \mathrm{M}$ DMED

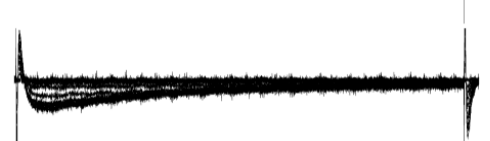

$1.0 \mu \mathrm{M}$ Yohimbine + $30 \mu \mathrm{M}$ DMED

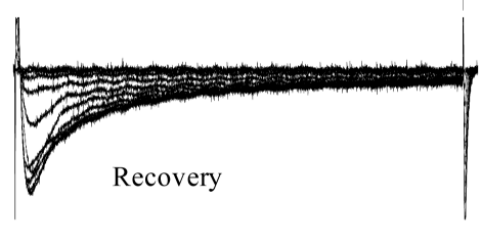

Figure 8 The inhibitory effects of DMED on hiPSC-CMs were mediated through $\alpha_{2}$-adrenoceptor-independent pathways. The $I_{\mathrm{Na}}$ was evoked by $300 \mathrm{~ms}$ depolarizing pulses of $-70 \mathrm{mV}$ to $0 \mathrm{mV}$ in $10 \mathrm{mV}$ increments from a holding potential of $-80 \mathrm{mV}$. The $I_{\mathrm{Ca}}$ was elicited by a series of depolarizing pulses from a holding potential of $-40 \mathrm{mV}$ to $+20 \mathrm{mV}$ in $10 \mathrm{mV}$ steps with a $300 \mathrm{~ms}$ pulse duration. Yohimbine did not affect the DMED-mediated effects on spontaneous action potential frequency (A), the $I_{\mathrm{Na}}(\mathrm{B})$, or the $I_{\mathrm{Ca}}(\mathrm{C})(\mathrm{P}>0.05$, n=5). hiPSC-CMs, human-induced pluripotent stem cell-derived cardiomyocytes; DMED, dexmedetomidine.

\section{$63.6 \% \pm 6.1 \%$ ) in hiPSC-CMs (Figure 10).}

\section{Discussion}

Following previously described protocols (25), about $80 \%$ of hiPSCs in this study were successfully differentiated into cardiomyocytes over 30 days. The differentiated cardiomyocytes were characterized by the presence of sarcomeric- $\alpha$-actinin and MLC2V, suggesting a ventricular phenotype. The action potential amplitude, maximal diastolic potential, $\mathrm{APD}_{90}$ (Figure 3), and frequency of contractions (Figure 2) recorded from the hiPSC-CMs cultured over 30 days were similar to that reported in previous studies $(21,26,27)$. These results indicated that the hiPSC-CMs generated by our induction and differentiation protocol were comparable to previous reports and the electrophysiological characteristics of the hiPSC-CMs used in the current study also agree with previous investigations. 
A

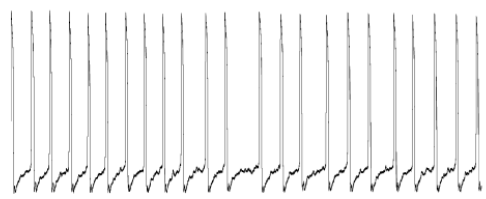

Control

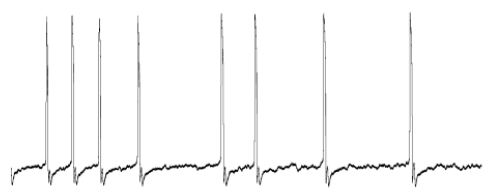

$10 \mu \mathrm{M}$ Idazoxan $+30 \mu \mathrm{M}$ DMED

B

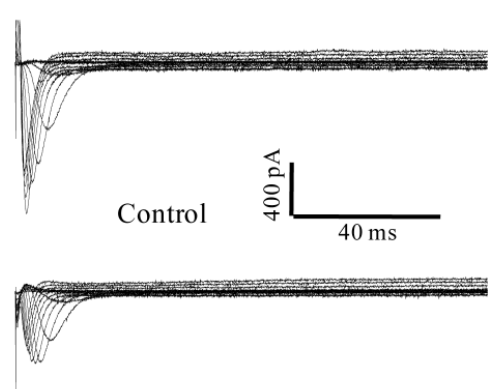

$30 \mu \mathrm{M}$ DMED
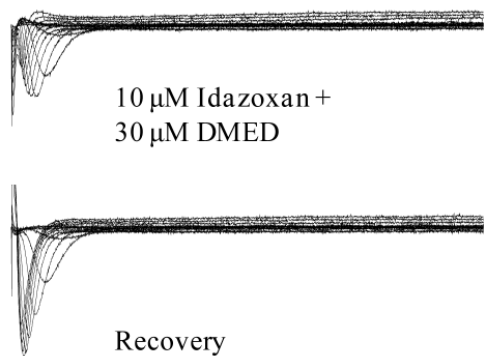
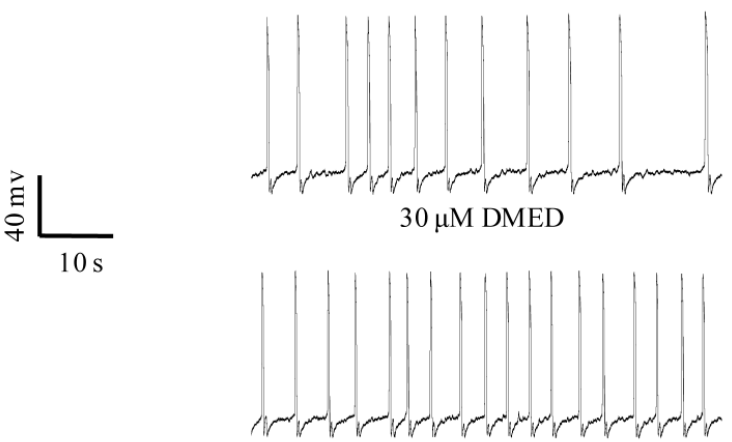

Recovery

C

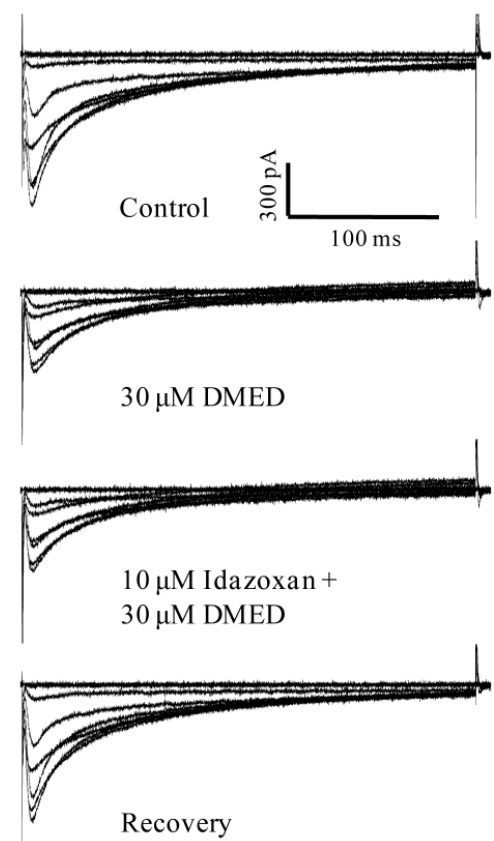

Figure 9 The inhibitory effects of DMED on hiPSC-CMs were mediated through imidazoline receptor-independent pathways. Idazoxan did not affect the DMED-mediated inhibition of spontaneous action potential frequency $(\mathrm{A})$, the $I_{\mathrm{Na}}(\mathrm{B})$, or the $I_{\mathrm{Ca}}(\mathrm{C})(\mathrm{P}>0.05, \mathrm{n}=5)$. hiPSCCMs, human-induced pluripotent stem cell-derived cardiomyocytes; DMED, dexmedetomidine.

The hiPSC-CMs generated in this study demonstrated ventricular-like spontaneous action potentials, and both the morphological and physiological characteristics were also suggestive of ventricular cardiomyocytes. However, the previous study has shown differences in the morphological and physiological characteristics between hiPSC-CMs and human ventricular myocytes (28). It has been widely reported that hiPSC-CMs structurally resemble human fetal ventricular cardiomyocytes. Human ventricular myocytes are large and cylindrical (approximately $150 \mu \mathrm{m} \times 10 \mu \mathrm{m}$ ), while hiPSC-CMs are smaller $(30 \mu \mathrm{m} \times 10 \mu \mathrm{m})(29)$. Also, human ventricular myocytes are bi- or multi-nucleated, whereas most hiPSC-CM are mono-nuclear. The extensive t-tubule network present in human ventricular myocytes is absent in hiPSC-CMs (30). Compared to mature ventricular myocytes, hiPSC-CMs exhibit reduced electrical excitability, impaired excitation-contraction coupling, and incomplete adrenergic sensitivity (28). However, it was reported that most ion channels present in healthy or diseased cardiomyocytes were also present in hiPSC-CMs, and in fact, some of them contribute to action potential performance and are regulated by adrenergic stimulation (31). 
A

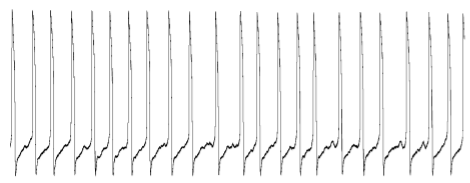

Control

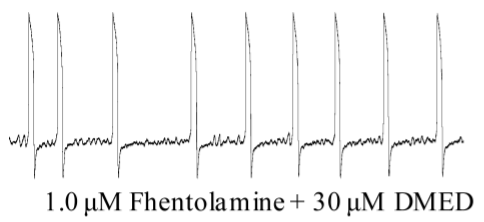

B

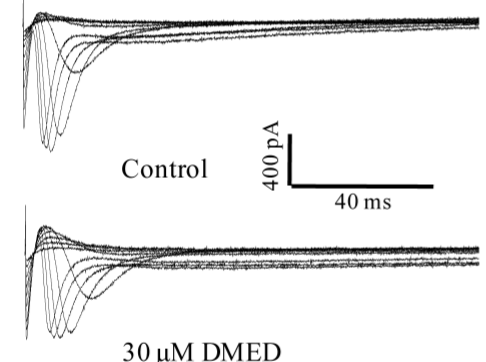

$30 \mu \mathrm{M}$ DMED

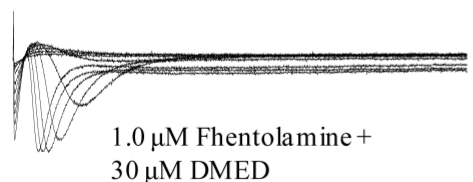

$30 \mu \mathrm{M}$ DMED

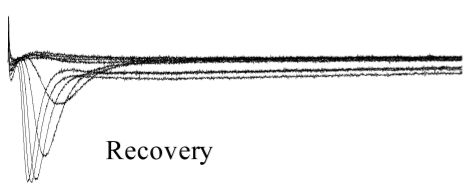

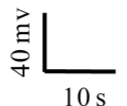
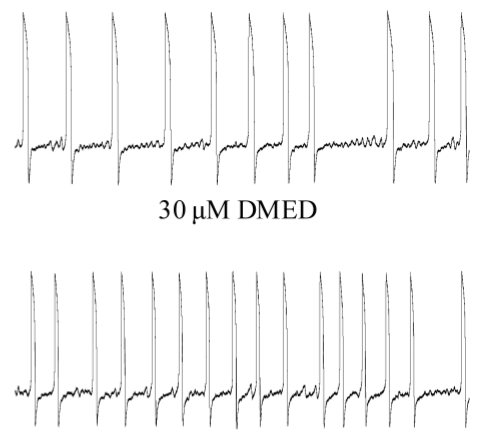

Recovery

C

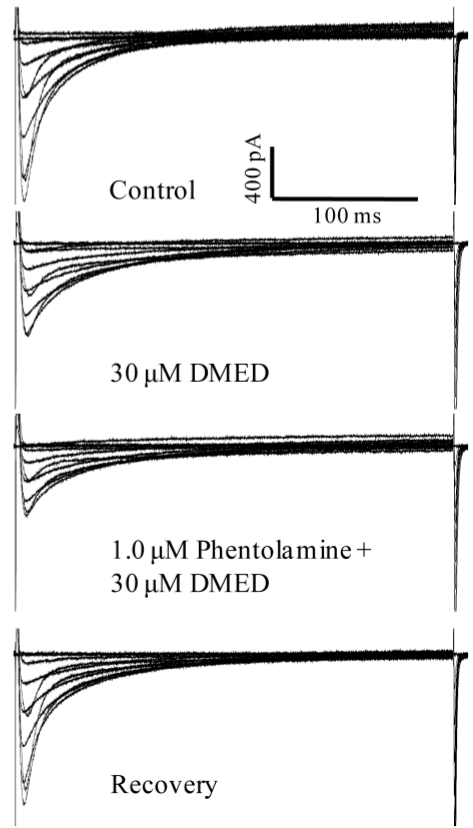

Figure 10 The inhibitory effects of DMED on hiPSC-CMs were mediated through $\alpha_{1}$-adrenoceptor-independent pathways. Phentolamine did not affect the inhibition of spontaneous action potential frequency $(\mathrm{A})$, the $I_{\mathrm{Na}}(\mathrm{B})$ or the $I_{\mathrm{Ca}}(\mathrm{C})$ mediated by $\mathrm{DMED}(\mathrm{P}>0.05$, n=5). hiPSC-CMs, human-induced pluripotent stem cell-derived cardiomyocytes; DMED, dexmedetomidine.

DMED inhibited the frequency of ventricular-like spontaneous action potentials and significantly prolonged the $\mathrm{APD}_{90}$ in a dose-dependent manner. DMED had a negligible effect on the $I_{\mathrm{f}}, I_{\mathrm{K} 1}$, and $I_{\mathrm{Kr}}$. However, DMED inhibited the $I_{\mathrm{Na}}$ and $I_{\mathrm{Ca}}$ of hiPSC-CMs, and this effect was independent of the $\alpha_{2}$-adrenoceptor, the imidazoline receptor, and the $\alpha_{1}$-adrenoceptor. The shapes of the activation and inactivation curves of the $\mathrm{Na}^{+}$and $\mathrm{Ca}^{2+}$ channels were not affected by DMED, but an effect was noted on time constant of activation and inactivation on these two channels.

The potential application of DMED in treating or preventing tachyarrhythmias has been reported, especially in the pediatric population (12). The antiarrhythmic effects of DMED are traditionally thought to be caused by the negative feedback modulation of catecholamine release through the activation of presynaptic $\alpha_{2}$-adrenoceptors expressed throughout the central nervous system and enhanced vagal activity $(32,33)$. However, recent in vitro experiments (34) showed that DMED attenuates L-calcium channel currents in dissociated ventricular myocytes from adult rat hearts and inhibits the firing rate of pacemaker cells from the sinoatrial nodes, indicating that DMED may have direct inhibitory effects on the myocardium. Our 
study further supports a DMED-mediated direct inhibitory effect on myocardial cells. We demonstrated that DMED dose-dependently inhibited the frequency of spontaneous ventricular-like action potentials and prolonged the $\mathrm{APD}_{90}$ in hiPSC-CMs without nerve innervations (Figures 2 and 3).

The $I_{\mathrm{f}}$ contributes to the spontaneous rhythmic activities in both the heart and the brain, and this rhythmic activity is in turn encoded by the HCN family of genes consisting of the HCN1-4 genes (35). Although the $I_{\mathrm{f}}$ current stemming from the sinoatrial node is essential for the onset and control of the heart rate (36), the $I_{\mathrm{f}}$ the current from hiPSC-CMs with ventricular-like action potentials did not mediate the inhibitory effects of DMED on the frequency of spontaneous action potentials. The $I_{\mathrm{K}}$ contributes to the plateau stage of cardiac ventricular cells. Although the $K_{\mathrm{r}}$ channel is a well-known nonspecific target of a broad range of drugs, the $I_{\mathrm{Kr}}$ current from hiPSC-CMs was not involved in the DMED-mediated prolongation of $\mathrm{APD}_{90}$. In this study, DMED did not affect the shape, amplitudes, and $\mathrm{I} / \mathrm{V}$ relationship curves of the $I_{\mathrm{K} 1}$ and $I_{\mathrm{Kr}}$, suggesting that DMED prolonged the $\mathrm{APD}_{90}$ of hiPSC-CMs through pathways independent of $I_{\mathrm{K} 1}$ and $I_{\mathrm{Kr}}$ (Figure 5).

The cardiac $I_{\mathrm{Na}}$ contributes to the action potential upstroke in ventricular cardiomyocytes. Our study demonstrated that DMED inhibited the amplitude of $I_{\mathrm{Na}}$, decreased the time constant of activation at the potential from -40 to $-20 \mathrm{mv}$, and increased the time constant of inactivation (Figure 6). Although a great number of studies have investigated the effects of DMED on $\mathrm{Na}^{+}$channels in different cells $(20,37,38)$, it is generally thought that the blockade of $\mathrm{Na}^{+}$channels is related to the high affinity of drugs to a site on the open, inactivated or resting state of $\mathrm{Na}^{+}$channels (39). Indeed, our previous study showed that DMED inhibited $I_{\mathrm{Na}}$ through preferential binding to inactivated $\mathrm{Na}^{+}$channels and produced a frequencydependent blockade of $\mathrm{Na}^{+}$channels in rat superior cervical ganglion (20). According to a study by Ross et al. (26), the average $I_{\mathrm{Na}}$ was about $6 \mathrm{nA}$, which was similar to our primary recording. To improve the quality of patch-clamp experiments and reduce voltage-clamp errors caused by large sodium currents, $100 \mathrm{mM} \mathrm{NaCl}$ in the extracellular solution for the $I_{\mathrm{Na}}$ recordings was replaced with $100 \mathrm{mM}$ choline chloride to reduce the peak values of the $I_{\mathrm{Na}}$.

In addition to the inhibition on the $I_{\mathrm{Na}}$, DMED also inhibited the amplitude of the $I_{\mathrm{Ca}}$, decreased the time constant of activation at potential $-20 \mathrm{mv}$, and increased the time constant of inactivation (Figure 7). Using rat dissociated ventricular myocytes, Zhao et al. showed that
DMED acted in a dose-dependent manner to inhibit the $I_{\mathrm{Ca}-\mathrm{L}}$ and slow down the activation process of $\mathrm{Ca}^{2+}$ channels but not the inactivation process. Additionally, DMED extended the recovery time from inactivation of the channels to reduce $\mathrm{Ca}^{2+}$ inward flow. This may contribute to the negative effects on myocardial contractility and cardiac electrical activity. Furthermore, the inhibition of the $I_{\mathrm{Ca}-\mathrm{L}}$ by DMED at a concentration of $10 \mathrm{ng} / \mathrm{mL}$ was partially reversed by yohimbine, suggesting that the inhibitory effect of DMED on the $I_{\mathrm{Ca}-\mathrm{L}}$ is partially associated with $\alpha_{2}$-adrenergic receptors (34). However, our study showed that DMED inhibited the $I_{\mathrm{Ca}}$ without affecting the calcium channel's activation and inactivation curves in hiPSC-CMs (Figure 7). Furthermore, the inhibitory effect of DMED on the $I_{\mathrm{Ca}}$ was not reversed by yohimbine (Figure 8), indicating that the inhibitory effect of DMED on the $I_{\mathrm{Ca}}$ in the hiPSCCMs was not associated with $\alpha_{2}$-adrenergic receptors. The differences between these two studies remain to be elucidated. According to a report by Yonemizu et al., there were no T-type $\mathrm{Ca}^{2+}$ channels detected in the hiPSC-CMs after 38-44 days of induction and differentiation (27).

Although $\alpha_{2}$-adrenoceptors, imidazoline receptors, and $\alpha_{1}$-adrenoceptors were detected in adult rat ventricular cardiomyocytes and hiPSC-CMs, the DMED-mediated inhibition of the action potential frequency, $\mathrm{Na}^{+}$channels, and $\mathrm{Ca}^{2+}$ channels were not reversed by yohimbine, idazoxan, or phentolamine, suggesting that the inhibitory effects caused by DMED in hiPSC-CMs were independent of the $\alpha_{2}$-adrenoceptor, the imidazoline receptor, and the $\alpha_{1}$-adrenoceptor. This contrasts with reports from Zhao et al., demonstrating that the inhibitory effects of DMED on the $I_{\mathrm{Ca}-\mathrm{L}}$ in rat ventricular myocytes were partially mediated by $\alpha_{2}$-adrenoceptors (34).

The explicit mechanisms by which DMED regulates ion channels have yet to be established in hiPSC-CMs. Although several methods have been adopted to enhance the maturation of hiPSC-CMs in vitro, hiPSC-CMs still demonstrate a fetal phenotype in structure and function $(40,41)$. As hiPSC-CMs do not perfectly imitate adult human cardiomyocytes, these differences may cause inaccurate disease modeling and drug screening results that may not precisely reflect the clinical setting. It is generally accepted that hiPSC-CMs could be utilized in a "case by case" manner to carefully screen patients' sensitivity and selectivity to certain drugs. Certain genetic compositions related to inherited genetic mutations or variations in the source cells also deserve careful consideration.

In conclusion, our results demonstrated that DMED 
induced inhibitory effects on the spontaneous action potential, the $I_{\mathrm{Na}}$, and the $I_{\mathrm{Ca}}$ of hiPSC-CMs, and these effects were not related to the $\alpha_{2}$-adrenoceptor, the imidazoline receptor, or the $\alpha_{1}$-adrenoceptor. Although further investigations are needed to elucidate the full effects of DMED on cardiomyocytes, our novel results suggest that the inhibitory effects of DMED on hiPSC-CMs may contribute to its antiarrhythmic effects.

\section{Acknowledgments}

The authors thank Professor Li Yanxin (Shanghai Children's Medical Center, Shanghai, China) for providing cell resources.

Funding: This study was sponsored by the National Natural Science Fund of China (81670372), the Shanghai Pujiang Program (18PJD031), the Shanghai Collaborative Innovation Center for Translational Medicine (TM201821), the National Key Research and Development Program of China (2018YFC2001904), the Biomedical Engineering Fund of Shanghai Jiao Tong University (YG2016MS28), the Shanghai Key Laboratory of Tissue Engineering, and the Pediatric Postgraduate Clinical Research Capacity Improvement Progress from the School of Medicine, Shanghai Jiao Tong University (EKKY2018003DGD).

\section{Footnote}

Reporting Checklist: The authors have completed the Materials Design Analysis Reporting (MDAR) checklist. Available at http://dx.doi.org/10.21037/atm-20-5898

Data Sharing Statement: Available at http://dx.doi. org/10.21037/atm-20-5898

Conflicts of Interest: All authors have completed the ICMJE uniform disclosure form (available at http://dx.doi. org/10.21037/atm-20-5898). The authors have no conflicts of interest to declare.

Ethical Statement: The authors are accountable for all aspects of the work in ensuring that questions related to the accuracy or integrity of any part of the work are appropriately investigated and resolved. All experiments were approved by the ethics committee of the Shanghai Children's Medical Center, Shanghai Jiao Tong University (SCMCIRB-W2020007).
Open Access Statement: This is an Open Access article distributed in accordance with the Creative Commons Attribution-NonCommercial-NoDerivs 4.0 International License (CC BY-NC-ND 4.0), which permits the noncommercial replication and distribution of the article with the strict proviso that no changes or edits are made and the original work is properly cited (including links to both the formal publication through the relevant DOI and the license). See: https://creativecommons.org/licenses/by-nc-nd/4.0/.

\section{References}

1. Chong JJ, Yang X, Don CW, et al. Human embryonicstem-cell-derived cardiomyocytes regenerate non-human primate hearts. Nature 2014;510:273-7.

2. Shiba Y, Gomibuchi T, Seto T, et al. Allogeneic transplantation of iPS cell-derived cardiomyocytes regenerates primate hearts. Nature 2016;538:388-91.

3. Gao L, Gregorich ZR, Zhu W, et al. Large Cardiac Muscle Patches Engineered From Human Induced-Pluripotent Stem Cell-Derived Cardiac Cells Improve Recovery From Myocardial Infarction in Swine. Circulation 2018;137:1712-30.

4. Sahara M, Santoro F, Chien KR. Programming and reprogramming a human heart cell. EMBO J 2015;34:710-38.

5. Burridge PW, Keller G, Gold JD, et al. Production of de novo cardiomyocytes: human pluripotent stem cell differentiation and direct reprogramming. Cell Stem Cell 2012;10:16-28.

6. Gibson JK, Yue Y, Bronson J, et al. Human stem cellderived cardiomyocytes detect drug-mediated changes in action potentials and ion currents. J Pharmacol Toxicol Methods 2014;70:255-67.

7. Asahi Y, Hamada T, Hattori A, et al. On-chip spatiotemporal electrophysiological analysis of human stem cell derived cardiomyocytes enables quantitative assessment of proarrhythmia in drug development. Sci Rep 2018;8:14536.

8. Sottas CE, Anderson BJ. Dexmedetomidine: the new all-in-one drug in paediatric anaesthesia? Curr Opin Anaesthesiol 2017;30:441-51.

9. Ebert TJ, Hall JE, Barney JA, et al. The effects of increasing plasma concentrations of dexmedetomidine in humans. Anesthesiology 2000;93:382-94.

10. Buckley MS, Agarwal SK, MacLaren R, et al. Adverse Hemodynamic Events Associated With Concomitant Dexmedetomidine and Propofol for Sedation in Mechanically Ventilated ICU Patients. J Intensive Care 
Med 2020;35:1536-45.

11. Keating GM. Dexmedetomidine: A Review of Its Use for Sedation in the Intensive Care Setting. Drugs 2015;75:1119-30.

12. Tobias JD, Chrysostomou C. Dexmedetomidine: antiarrhythmic effects in the pediatric cardiac patient. Pediatr Cardiol 2013;34:779-85.

13. Weerink MAS, Struys MMRF, Hannivoort LN, et al. Clinical Pharmacokinetics and Pharmacodynamics of Dexmedetomidine. Clin Pharmacokinet 2017;56:893-913.

14. Correa-Sales C, Rabin BC, Maze M. A hypnotic response to dexmedetomidine, an alpha 2 agonist, is mediated in the locus coeruleus in rats. Anesthesiology. 1992;76:948-52.

15. Chiu TH, Chen MJ, Yang YR, et al. Action of dexmedetomidine on rat locus coeruleus neurones: intracellular recording in vitro. Eur J Pharmacol 1995;285:261-8.

16. Pan X, Zhang Z, Huang YY, et al. Electrophysiological Effects of Dexmedetomidine on Sinoatrial Nodes of Rabbits. Acta Cardiol Sin 2015;31:543-9.

17. Knaus A, Zong X, Beetz N, et al. Direct inhibition of cardiac hyperpolarization-activated cyclic nucleotidegated pacemaker channels by clonidine. Circulation 2007;115:872-80.

18. Gu H, Huang X, Xu J, et al. Optimizing the method for generation of integration-free induced pluripotent stem cells from human peripheral blood. Stem Cell Res Ther 2018;9:163.

19. Takamatsu I, Iwase A, Ozaki M, et al. Dexmedetomidine reduces long-term potentiation in mouse hippocampus. Anesthesiology 2008;108:94-102.

20. Yang L, Tang J, Dong J, et al. Alpha2-adrenoceptorindependent inhibition of acetylcholine receptor channel and sodium channel by dexmedetomidine in rat superior cervical ganglion neurons. Neuroscience 2015;289:9-18.

21. Ma J, Guo L, Fiene SJ, et al. High purity humaninduced pluripotent stem cell-derived cardiomyocytes: electrophysiological properties of action potentials and ionic currents. Am J Physiol Heart Circ Physiol 2011;301:H2006-17.

22. Verkerk AO, Wilders R. Pacemaker activity of the human sinoatrial node: effects of $\mathrm{HCN} 4$ mutations on the hyperpolarization-activated current. Europace 2014;16:384-95.

23. DiFrancesco D. The role of the funny current in pacemaker activity. Circ Res 2010;106:434-46.

24. Tamargo J, Valenzuela C, Delpón E. New insights into the pharmacology of sodium channel blockers. Eur Heart J.
1992;13 Suppl F:2-13.

25. Lian X, Hsiao C, Wilson G, et al. Robust cardiomyocyte differentiation from human pluripotent stem cells via temporal modulation of canonical Wnt signaling. Proc Natl Acad Sci U S A 2012;109:E1848-57.

26. Ross GR, Rizvi F, Emelyanova L, et al. Prolonged post-differentiation culture influences the expression and biophysics of $\mathrm{Na}(+)$ and $\mathrm{Ca}(2+)$ channels in induced pluripotent stem cell-derived ventricular-like cardiomyocytes. Cell Tissue Res 2019;378:59-66.

27. Yonemizu S, Masuda K, Kurata Y, et al. Inhibitory effects of class I antiarrhythmic agents on $\mathrm{Na}(+)$ and $\mathrm{Ca}(2+)$ currents of human iPS cell-derived cardiomyocytes. Regen Ther 2019; 10:104-11.

28. Robertson C, Tran DD, George SC. Concise review: maturation phases of human pluripotent stem cell-derived cardiomyocytes. Stem Cells 2013;31:829-37.

29. Snir M, Kehat I, Gepstein A, et al. Assessment of the ultrastructural and proliferative properties of human embryonic stem cell-derived cardiomyocytes. Am J Physiol Heart Circ Physiol 2003;285:H2355-63.

30. Lieu DK, Liu J, Siu C, et al. Absence of Transverse Tubules Contributes to Non-Uniform Ca2+ Wavefronts in Mouse and Human Embryonic Stem Cell-Derived Cardiomyocytes. Stem Cells Dev 2009;18:1493-500.

31. Zhao Z, Lan H, El-Battrawy I, et al. Ion Channel Expression and Characterization in Human Induced Pluripotent Stem Cell-Derived Cardiomyocytes. Stem Cells Int 2018;2018:6067096.

32. Hayashi Y, Sumikawa K, Maze M, et al. Dexmedetomidine prevents epinephrine-induced arrhythmias through stimulation of central alpha 2 adrenoceptors in halothaneanesthetized dogs. Anesthesiology. 1991;75:113.

33. Kamibayashi T, Hayashi Y, Mammoto T, et al. Role of the vagus nerve in the antidysrhythmic effect of dexmedetomidine on halothane/epinephrine dysrhythmias in dogs. Anesthesiology. 1995;83:992-9.

34. Zhao J, Zhou CL, Xia ZY, et al. Effects of Dexmedetomidine on L-Type Calcium Current in Rat Ventricular Myocytes. Acta Cardiol Sin 2013;29:175-80.

35. Morikawa K, Bahrudin U, Miake J, et al. Identification, isolation and characterization of HCN4-positive pacemaking cells derived from murine embryonic stem cells during cardiac differentiation. Pacing Clin Electrophysiol 2010;33:290-303.

36. Biel M, Wahl-Schott C, Michalakis S, et al. Hyperpolarization-activated cation channels: from genes to function. PHYSIOL REV 2009;89:847-85. 


\section{Page 18 of 18}

37. Im ST, Jo YY, Han G, et al. Dexmedetomidine Inhibits Voltage-Gated Sodium Channels via alpha2-Adrenoceptors in Trigeminal Ganglion Neurons. Mediators Inflamm 2018;2018:1782719.

38. Oda A, Iida H, Tanahashi S, et al. Effects of alpha2adrenoceptor agonists on tetrodotoxin-resistant $\mathrm{Na}+$ channels in rat dorsal root ganglion neurons. Eur J Anaesthesiol 2007;24:934-41.

39. Xie XM, Garthwaite J. State-dependent inhibition of $\mathrm{Na}+$ currents by the neuroprotective agent 619C89 in

Cite this article as: Yang L, Gong Y, Tan Y, Wu L, Witman N, Zheng J, Zhang J, Fu W, Wang W. Dexmedetomidine exhibits antiarrhythmic effects on human-induced pluripotent stem cellderived cardiomyocytes through a $\mathrm{Na} / \mathrm{Ca}$ channel-mediated mechanism. Ann Transl Med 2021;9(5):399. doi: 10.21037/atm20-5898

\section{Yang et al. Direct negative chronotropic effects of DMED}

rat hippocampal neurons and in a mammalian cell line expressing rat brain type IIA $\mathrm{Na}+$ channels. Neuroscience. 1996;73:951-62.

40. Yang X, Pabon L, Murry CE. Engineering adolescence: maturation of human pluripotent stem cell-derived cardiomyocytes. Circ Res 2014;114:511-23.

41. Zhu R, Blazeski A, Poon E, et al. Physical developmental cues for the maturation of human pluripotent stem cellderived cardiomyocytes. Stem Cell Res Ther 2014;5:117. 